\title{
Stability of $\mathrm{AdS}_{p} \times \mathrm{M}_{q}$ compactifications without supersymmetry
}

\author{
Oliver DeWolfe, ${ }^{1}$ Daniel Z. Freedman, ${ }^{2,3}$ Steven S. Gubser, ${ }^{4,5}$ Gary T. Horowitz, ${ }^{1,6}$ and Indrajit Mitra ${ }^{5}$ \\ ${ }^{1}$ Institute for Theoretical Physics, UCSB, Santa Barbara, California 93106 \\ ${ }^{2}$ Caltech-USC Center for Theoretical Physics, USC, Los Angeles, California 90089 \\ ${ }^{3}$ Department of Mathematics and Center for Theoretical Physics, Massachusetts Institute of Technology, \\ Cambridge, Massachusetts 02139 \\ ${ }^{4}$ Lauritsen Laboratory of Physics, Caltech, Pasadena, California 91125 \\ ${ }^{5}$ Joseph Henry Laboratories, Princeton University, Princeton, New Jersey 08544 \\ ${ }^{6}$ Department of Physics, UCSB, Santa Barbara, California 93106
}

(Received 22 September 2001; published 4 March 2002)

\begin{abstract}
We study the stability of Freund-Rubin compactifications, $\operatorname{AdS}_{p} \times \mathrm{M}_{q}$, of $(p+q)$-dimensional gravity theories with a $q$-form field strength and no cosmological term. We show that the general $\operatorname{AdS}_{p} \times \mathrm{S}^{q}$ vacuum is classically stable against small fluctuations, in the sense that all modes satisfy the Breitenlohner-Freedman bound. In particular, the compactifications used in the recent discussion of the proposed bosonic M theory are perturbatively stable. Our analysis treats all modes arising from the graviton and the $q$ form, and is completely independent of supersymmetry. From the masses of the linearized perturbations, we obtain the dimensions of some operators in possible holographic dual CFT's. Solutions with more general compact Einstein spaces need not be stable, and in particular $\operatorname{AdS}_{p} \times \mathrm{S}^{n} \times \mathrm{S}^{q-n}$ is unstable for $q<9$ but is stable for $q \geqslant 9$. We also study the $\mathrm{AdS}_{4} \times \mathrm{S}^{6}$ compactification of massive type IIA supergravity, which differs from the usual Freund-Rubin compactification in that there is a cosmological term already in ten dimensions. This nonsupersymmetric vacuum is unstable.
\end{abstract}

DOI: 10.1103/PhysRevD.65.064033

PACS number(s): 04.50. $+\mathrm{h}$

\section{INTRODUCTION}

The discovery of the AdS/CFT correspondence [1-3] (for a review see [4]) has led to renewed interest in the stability of geometries of the form $\operatorname{AdS}_{p} \times \mathrm{M}_{q}$ where $\mathrm{AdS}_{p}$ is anti-de Sitter spacetime and $\mathrm{M}_{q}$ is an Einstein space with positive Ricci tensor. Solutions of this type with a $q$-form field strength on $\mathrm{M}_{q}$ were first considered in higher dimensional supergravity theories by Freund and Rubin [5]. Because of the negative curvature of AdS, perturbative stability does not require the absence of all tachyonic modes. Instead, as Breitenlohner and Freedman (BF) first showed, scalars with $m^{2}<0$ may appear as long as their masses do not fall below a bound set by the curvature scale of AdS [6]. The issue of stability is important for understanding a possible dual conformal field theory (CFT) description. For stable solutions, the spectrum of masses directly yields the dimensions of certain operators in such a CFT. Unstable solutions can still have a dual CFT description [7], but the physics is clearly very different.

It is well known that for the standard ten and eleven dimensional maximally supersymmetric supergravity (SUGRA) theories, 11D SUGRA on $\mathrm{AdS}_{4} \times \mathrm{S}^{7}$ or $\mathrm{AdS}_{7} \times \mathrm{S}^{4}$ and type IIB SUGRA on $\mathrm{AdS}_{5} \times \mathrm{S}^{5}$ are all stable. However, these solutions are all supersymmetric (SUSY), and simple nonsupersymmetric vacua such as $\mathrm{AdS}_{4} \times \mathrm{M}_{n}$ $\times \mathrm{M}_{7-n}[8]$ and $\mathrm{AdS}_{7} \times \mathrm{S}^{2} \times \mathrm{S}^{2}[9]$ are known to be unstable. Furthermore, the SUSY examples have modes which either saturate the BF bound, or are very close to saturating it. This raises the question of the role that SUSY plays in ensuring stability of vacua of this type. (For earlier discussions of this question see e.g., [8-10].) This issue is of particular interest in light of the recent proposal of bosonic $\mathrm{M}$ theory [11], a 27-dimensional theory which was hypothesized to appear as the strong-coupling limit of the bosonic string. Its low energy limit is assumed to be gravity coupled to a four-form field strength, which admits solutions of the form $\mathrm{AdS}_{4} \times \mathrm{S}^{23}$ and $\operatorname{AdS}_{23} \times \mathrm{S}^{4}$. It was suggested that with these boundary conditions, bosonic $\mathrm{M}$ theory might be holographically described by a $(2+1)$ - or $(21+1)$-dimensional CFT. Thus, it is important to determine whether these solutions are stable.

One argument for the stability of $\mathrm{AdS}_{4} \times \mathrm{S}^{23}$ [11] and more generally $\operatorname{AdS}_{p} \times \mathrm{S}^{q}$ is that these backgrounds are the near-horizon geometries of extremal black branes. However this is not completely satisfying for two reasons. First, although we expect extremal black branes to be stable, the appropriate positive mass theorem (stating roughly $M \geqslant Q$ ) has never been proven. ${ }^{1}$ Second, as we will discuss later, one can construct extremal black brane solutions with unstable near horizon geometry by placing branes at the apex of appropriate cones. So, one needs to examine stability directly.

In this paper, we study the stability of general solutions of the form $\operatorname{AdS}_{p} \times \mathrm{M}_{q}$ in a theory of gravity coupled to a $q$-form field strength. When one expands the field equations to linear order, there are several types of modes. Some immediately decouple from the others, while the rest mix and must be diagonalized. A priori, since the fundamental fields in $p+q$ dimensions are massless, and adding dependence on $\mathrm{M}_{q}$ should increase the mass, one might expect that the modes that do not mix should always be stable. Masses violating the BF bound might be expected, however, to arise in diagonalizing the coupled fluctuations-indeed, this is the

\footnotetext{
${ }^{1}$ Interestingly enough, if one tries to adapt Witten's spinorial approach, one succeeds only in the SUSY cases [12].
} 
origin of the modes that saturate or come very near to saturating the $\mathrm{BF}$ bound in the SUSY examples, so one might think that the absence of supersymmetry could push them over the edge.

Surprisingly, this is not what we find. It turns out that for any $p$ and $q$ and any Einstein space $\mathbf{M}_{q}$, the coupled modes are always stable. Moreover, for $\mathrm{S}^{q}$ the lowest mass either saturates ( $q$ odd) or almost saturates ( $q$ even) the BF bound. This is not to say, however, that an arbitrary $\operatorname{AdS}_{p} \times \mathrm{M}_{q}$ background is stable. The dangerous mode turns out to be an unmixed scalar coming from the transverse, traceless metric perturbation on $\mathrm{M}_{q}$. This is the only mode which is sensitive to the choice of Einstein manifold $\mathbf{M}_{q}$. If $\mathbf{M}_{q}$ is the round sphere $S^{q}$, it is easy to show that this mode is stable. In particular, the spacetimes of interest for bosonic M theory, $\mathrm{AdS}_{4} \times \mathrm{S}^{23}$ and $\mathrm{AdS}_{23} \times \mathrm{S}^{4}$, are stable. However, if $\mathrm{M}_{q}$ $=\mathrm{M}_{n} \times \mathrm{M}_{q-n}$ and $q<9$, there is a mass violating the $\mathrm{BF}$ bound, corresponding to a mode which makes one factor grow while the other shrinks. This generalizes the instabilities of $\operatorname{AdS}_{4} \times \mathrm{M}_{n} \times \mathrm{M}_{7-n}$ and $\mathrm{AdS}_{7} \times \mathrm{S}^{2} \times \mathrm{S}^{2}$, but also shows that this instability is limited to low dimensions. For $q \geqslant 9, \quad \operatorname{AdS}_{p} \times \mathrm{S}^{n} \times \mathrm{S}^{q-n}$ can be shown to be stable. The significance of the critical dimension $q=9$ is not clear; it is sufficiently large that stable products cannot be realized in superstring or M theory.

The massive type IIA supergravity has a nonsupersymmetric vacuum of the form $\operatorname{AdS}_{4} \times S^{6}$ [13], whose stability, to our knowledge, has never been investigated. We also study this case and show that the solution is unstable, with two modes violating the $\mathrm{BF}$ bound. To our knowledge, this is the first example of a theory where the product of AdS and a round sphere is unstable. The analysis is more involved here since there is a dilaton which mixes with some of the other modes, further complicating the coupled sector. Instabilities for more general $\mathrm{AdS}_{4} \times \mathrm{M}_{6}$ can arise in several ways, but we show in particular that they do occur for $\mathrm{AdS}_{4} \times \mathrm{S}^{n} \times \mathrm{S}^{6-n}$.

There is a vast literature on Kaluza-Klein theories, much of it in the context of higher dimensional supergravity, including a comprehensive review [14]. Our treatment of the harmonic analysis of fluctuations about $\operatorname{AdS}_{p} \times \mathrm{M}_{q}$ is most closely modeled on [15-17], and we have also consulted [8] and [18]. In Sec. II we present the general $\mathrm{AdS}_{p} \times \mathrm{M}_{q}$ background solution. The harmonic expansions for fluctuations and their linear equations of motion are discussed in Sec. III. The mass spectra of the various fluctuations are analyzed in Secs. IV-VIII. The more complicated case of massive type IIA supergravity is discussed in Sec. IX. The $\operatorname{AdS}_{p}$ mass spectra determine the dimensions of operators in hypothetical $\mathrm{CFT}_{p-1}$ dual field theories, and this is discussed in Sec. X. In Sec. XI, we show that for some of the unstable cases, the total energy (in the full nonlinear theory) is unbounded from below. We also speculate on the implications of our results for the stability of certain extremal black brane solutions. Conventions and properties of various differential operators are collected in the Appendix.

\section{FREUND-RUBIN BACKGROUNDS}

We start by considering classical $D=p+q$ dimensional gravity theory coupled to a $q$-form field strength. The action is given by

$$
S=\int d^{p} x d^{q} y \sqrt{-g}\left(R-\frac{1}{2 q !} F_{q}^{2}\right),
$$

which leads to the equations of motion

$$
\begin{aligned}
R_{M N}= & \frac{1}{2(q-1) !} F_{M P_{2} \cdots P_{q}} F_{N}{ }^{P_{2} \cdots P_{q}} \\
& -\frac{(q-1)}{2(D-2) q !} g_{M N} F_{q}^{2}, \\
d * F_{q}= & 0 .
\end{aligned}
$$

This theory supports a Freund-Rubin solution with the product metric

$$
d s^{2}=d s_{\mathrm{AdS}_{p}}^{2}+d s_{M_{q}}^{2},
$$

describing a product of $p$-dimensional anti-de Sitter space with an Einstein manifold:

$$
\begin{aligned}
& R_{\mu \nu}=-\frac{(p-1)}{L^{2}} g_{\mu \nu}, \\
& R_{\alpha \beta}=\frac{(q-1)}{R^{2}} g_{\alpha \beta},
\end{aligned}
$$

and a background field strength on the compact space:

$$
F_{q}=c \operatorname{vol}_{M_{q}}
$$

We use $M, N, \ldots$ for indices on the full $D$-dimensional spacetime, while $\mu, \nu, \ldots$ are indices on $\operatorname{AdS}$ and $\alpha, \beta, \ldots$ are indices on $M_{q}$. The equations of motion (2.2), (2.3) relate the length scales $L$ and $R$ and the constant $c$ :

$$
\begin{aligned}
c^{2} & =\frac{2(D-2)(q-1)}{(p-1) R^{2}}, \\
L & =\frac{p-1}{q-1} R .
\end{aligned}
$$

In the following six sections we shall study fluctuations of $g_{M N}$ and $F_{q}$ around this background. Among other things, we will conclude that the background is stable against these fluctuations when $\mathrm{M}_{q}=\mathrm{S}^{q}$, for arbitrary $p>2$ and $q>1$. If one wishes to embed the action (2.1) in a larger theory with additional fields, stability must be verified separately for the new modes. However let us note that the most tachyonic modes in the well-studied vacua of ten- and elevendimensional supergravities generally come from precisely the fields which support the solution. Thus, when these most "dangerous" modes come out stable, it suggests that the background is probably stable against all fluctuations. 


\section{LINEARIZED EQUATIONS OF MOTION}

\section{A. Fluctuations}

We are interested in studying the stability of linearized fluctuations around the background (2.4), (2.7). As we have discussed, anti-de Sitter space is stable even in the presence of tachyonic scalar fields, as long as their masses do not violate the Breitenlohner-Freedman bound:

$$
m^{2} L^{2} \geqslant-\frac{(p-1)^{2}}{4} .
$$

The possibility that some tachyons could be acceptable in $\mathrm{AdS}_{4}$ was first pointed out by Breitenlohner and Freedman [6], and extended to $\mathrm{AdS}_{p}$ by [19]. See also [20,21] for early developments of this idea.

We consider the linearized fluctuations

$$
\begin{gathered}
\delta g_{\mu \nu}=h_{\mu \nu}=H_{\mu \nu}-\frac{1}{p-2} g_{\mu \nu} h_{\alpha}^{\alpha}, \\
\delta g_{\mu \alpha}=h_{\mu \alpha}, \quad \delta g_{\alpha \beta}=h_{\alpha \beta}, \\
\delta A_{q-1}=a_{q-1}, \quad \delta F_{q} \equiv f_{q}=d a_{q-1},
\end{gathered}
$$

where we have defined a standard linearized Weyl shift on $h_{\mu \nu}$ in Eq. (3.2), and $F_{q}=d A_{q-1}$. It will be useful to decompose $H_{\mu \nu}$ and $h_{\alpha \beta}$ into trace and traceless parts:

$$
H_{\mu \nu}=H_{(\mu \nu)}+\frac{1}{p} g_{\mu \nu} H_{\rho}^{\rho}, \quad h_{\alpha \beta}=h_{(\alpha \beta)}+\frac{1}{q} g_{\alpha \beta} h_{\gamma}^{\gamma},
$$

where $g^{\mu \nu} H_{(\mu \nu)}=g^{\alpha \beta} h_{(\alpha \beta)}=0$. To $\left(\right.$ mostly $\left.{ }^{2}\right)$ fix the internal diffeomorphisms and gauge freedom, we impose the de Donder-type gauge conditions

$$
\nabla^{\alpha} h_{(\alpha \beta)}=\nabla^{\alpha} h_{\alpha \mu}=0,
$$

as well as the Lorentz-type conditions

$$
\begin{aligned}
\nabla^{\alpha} a_{\alpha \beta_{2} \ldots \beta_{q-1}} & =\nabla^{\alpha} a_{\alpha \beta_{2} \ldots \beta_{q-2} \mu}=\ldots=\nabla^{\alpha} a_{\alpha \mu_{2} \ldots \mu_{q-1}} \\
& =0 .
\end{aligned}
$$

A generic gauge potential $a_{\alpha_{1} \ldots \alpha_{n} \mu_{n+1} \ldots \mu_{q-1}}$, viewed as an $n$-form on $\mathrm{M}_{q}$ with additional $\mathrm{AdS}_{p}$ indices, can be expanded as the sum of an exact, a co-exact and a harmonic form on $\mathrm{M}_{q}$ by the Hodge decomposition theorem. The Lorentz conditions (3.6), which state that the form is co-exact, require the exact form in the decomposition to vanish, and hence the potentials can be expanded as co-exact forms (curls) and harmonic forms:

\footnotetext{
${ }^{2}$ In addition to unfixed $p$-dimensional diffeomorphisms and gauge transformations, extra conformal diffeomorphisms remain on $S^{q}$. These are related to the elimination of a $k=1$ mode in the coupled scalar sector, as in Sec. IV; for a discussion, see [16].
}

$$
\begin{aligned}
& a_{\beta_{1} \ldots \beta_{n} \mu_{n+1} \mu_{q-1}} \\
& =\epsilon_{\beta_{1} \ldots \beta_{n}}^{\alpha_{1} \alpha_{2} \ldots \alpha_{q-n}} \nabla_{\alpha_{1}} b_{\alpha_{2} \ldots \alpha_{q-n} \mu_{n+1} \ldots \mu_{q-1}} \\
& \quad+\beta_{\beta_{1} \ldots \beta_{n} \mu_{n+1} \mu_{q-1}}^{\text {harm }}
\end{aligned}
$$

When the compact space is an $\mathrm{S}^{q}$ there are no nontrivial harmonic forms, but they can appear for other $\mathbf{M}_{q}$. In a compact notation, we may write Eqs. (3.6) and (3.7) as

$$
d_{q} *_{q} a=0 \rightarrow a=*{ }_{q} d_{q} b+\beta^{h a r m},
$$

where $d_{q}$ and $*_{q}$ are the exterior derivative and Hodge dual with respect to the $M_{q}$ space only.

With these gauge choices, we may expand the fluctuations in spherical harmonics as

$$
\begin{aligned}
& H_{(\mu \nu)}(x, y)=\sum_{I} H_{(\mu \nu)}^{I}(x) Y^{I}(y), \\
& H_{\mu}^{\mu}(x, y)=\sum_{I} H^{I}(x) Y^{I}(y), \\
& h_{(\alpha \beta)}(x, y)=\sum_{I} \phi^{I}(x) Y_{(\alpha \beta)}^{I}(y), \\
& h_{\alpha}^{\alpha}(x, y)=\sum_{I} \pi^{I}(x) Y^{I}(y), \\
& h_{\mu \alpha}(x, y)=\sum_{I} B_{\mu}^{I}(x) Y_{\alpha}^{I}(y) \text {, } \\
& a_{\beta_{1} \ldots \beta_{q-1}}=\sum_{I} b^{I}(x) \epsilon_{\beta_{1} \ldots \beta_{q-1}}^{\alpha} \nabla_{\alpha} Y^{I}(y), \\
& a_{\mu \beta_{2} \ldots \beta_{q-1}}=\sum_{I} b_{\mu}^{I}(x) \epsilon_{\beta_{2} \ldots \beta_{q-1}}^{\alpha \beta} \nabla_{[\alpha} Y_{\beta]}^{I}(y) \\
& +\sum_{h} \beta_{\mu}^{h}(x) \epsilon_{\beta_{2} \ldots \beta_{q-1}}^{\alpha \beta} Y_{[\alpha \beta]}^{h}, \\
& a_{\mu_{1} \ldots \mu_{q-1}}=\sum_{I} b_{\mu_{1} \ldots \mu_{q-1}}^{I}(x) Y^{I}(y),
\end{aligned}
$$

where $I$ in each case is a generic label running over the possible spherical harmonics of the appropriate tensor type, and $h=1 \ldots b^{n}\left(\mathrm{M}_{q}\right)$ runs over the harmonic $n$-forms on $\mathbf{M}_{q}$ for the gauge field with $(n-1) \mathrm{AdS}_{p}$ indices. We have not included a term $\beta(x)$ in Eq. (3.12) since compact Riemannian Einstein spaces with positive curvature cannot possess harmonic one-forms; this is proved in the Appendix. We will also find it convenient to define

$$
b(x, y) \equiv \sum_{I} b^{I}(x) Y^{I}(y), \quad b_{\mu \alpha}(x, y) \equiv \sum_{I} b_{\mu}^{I}(x) Y_{\alpha}^{I}(y) .
$$




\section{B. Einstein equations and coupled form equations}

We now consider the Einstein equations to linear order in fluctuations, as well as the form equations that mix with the graviton; the uncoupled form equations will be treated in Sec. VII. We use the following notation: $\square_{x} \equiv g^{\mu \nu} \nabla_{\mu} \nabla_{\nu}$, $\square_{y} \equiv g^{\alpha \beta} \nabla_{\alpha} \nabla_{\beta}$, and $\max B_{\mu} \equiv \square_{x} B_{\mu}-\nabla^{\nu} \nabla_{\mu} B_{\nu}$ is the Maxwell operator acting on vectors on $\mathrm{AdS}_{q}$. Additionally, $\Delta_{y}$ $\equiv-\left(d_{q}^{\dagger} d_{q}+d_{q} d_{q}^{\dagger}\right)$ is the Laplacian ${ }^{3}$ acting on differential forms on $M_{q}$; for vectors, the explicit form is $\Delta_{y} Y_{\alpha}$

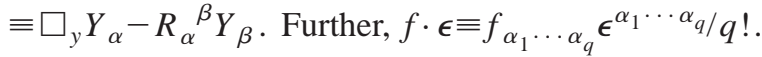

For convenience, we present the linearized Ricci tensor in our conventions:

$$
\begin{aligned}
R_{M N}^{(1)}= & -\frac{1}{2}\left[\left(\square_{x}+\square_{y}\right) h_{M N}+\nabla_{M} \nabla_{N} h_{P}^{P}-\nabla_{M} \nabla^{P} h_{P N}\right. \\
& \left.-\nabla_{N} \nabla^{P} h_{P M}-2 R_{M P Q N} h^{P Q}-R_{M}{ }^{P} h_{N P}-R_{N}{ }^{P} h_{M P}\right] .
\end{aligned}
$$

We employ Einstein's equations in their Ricci form, $R_{M N}$ $=\bar{T}_{M N}$ with $\bar{T}_{M N} \equiv T_{M N}+g_{M N} T_{P}^{P} /(2-D)$. For $R_{\mu \nu}$ we find

$$
\begin{aligned}
R_{\mu \nu}{ }^{(1)}= & -\frac{1}{2}\left[\left(\square_{x}+\square_{y}\right)\left(H_{\mu \nu}-\frac{1}{p-2} g_{\mu \nu} h_{\gamma}^{\gamma}\right)\right. \\
& +\nabla_{\mu} \nabla_{\nu}\left(H_{\rho}^{\rho}-\frac{2}{p-2} h_{\gamma}^{\gamma}\right) \\
& -\nabla_{\mu} \nabla^{\rho}\left(H_{\rho \nu}-\frac{1}{p-2} g_{\rho \nu} h_{\gamma}^{\gamma}\right) \\
& -\nabla_{\nu} \nabla^{\rho}\left(H_{\rho \mu}-\frac{1}{p-2} g_{\rho \mu} h_{\gamma}^{\gamma}\right) \\
& -2 R_{\mu \rho \sigma \nu}\left(H^{\rho \sigma}-\frac{1}{p-2} g^{\rho \sigma} h_{\gamma}^{\gamma}\right) \\
& -R_{\mu}{ }^{\rho}\left(H_{\rho \nu}-\frac{1}{p-2} g_{\rho \nu} h_{\gamma}^{\gamma}\right) \\
& \left.-R_{\nu}{ }^{\rho}\left(H_{\rho \mu}-\frac{1}{p-2} g_{\rho \mu} h_{\gamma}^{\gamma}\right)\right]
\end{aligned}
$$

which must be equal to

$$
\begin{aligned}
\bar{T}_{\mu \nu}^{(1)}= & -\frac{c^{2}(q-1)}{2(D-2)} h_{\mu \nu}-\frac{q(q-1) c^{2}}{2(D-2) q !} g_{\mu \nu}\left(-h^{\alpha \beta}\right) \\
& \times \epsilon_{\alpha \gamma_{2} \cdots \gamma_{q}} \epsilon_{\beta}^{\gamma_{2} \cdots \gamma_{q}}-\frac{c(q-1)}{(D-2)} g_{\mu \nu}(f \cdot \epsilon),
\end{aligned}
$$

resulting in the equation

${ }^{3}$ The negative sign is standard in the Kaluza-Klein literature.

$$
\begin{aligned}
& -\frac{1}{2}\left[\left(\square_{x}+\square_{y}\right) H_{\mu \nu}+\nabla_{\mu} \nabla_{\nu} H_{\rho}^{\rho}-\nabla_{\mu} \nabla^{\rho} H_{\rho \nu}-\nabla_{\nu} \nabla^{\rho} H_{\rho \mu}\right. \\
& \left.\quad-2 R_{\mu \rho \sigma \nu} H^{\rho \sigma}-R_{\mu}{ }^{\rho} H_{\rho \nu}-R_{\nu}{ }^{\rho} H_{\rho \mu}\right] \\
& \quad+\frac{1}{2(p-2)} g_{\mu \nu}\left(\square_{x}+\square_{y}\right) h_{\gamma}^{\gamma}-\frac{(q-1)^{2}}{(p-2) R^{2}} g_{\mu \nu} h_{\gamma}^{\gamma} \\
& \quad+\frac{(q-1)^{2}}{(p-1) R^{2}} H_{\mu \nu}+\frac{q-1}{D-2} g_{\mu \nu} \square_{y} c b=0 .
\end{aligned}
$$

For linearized $R_{\mu \alpha}$, we find

$$
\begin{aligned}
R_{\mu \alpha}^{(1)} & =-\frac{1}{2}\left[\square_{x} h_{\mu \alpha}-\nabla_{\mu} \nabla^{\nu} h_{\nu \alpha}-R_{\mu}{ }^{\nu} h_{\nu \alpha}+\square_{y} h_{\mu \alpha}-R_{\alpha}{ }^{\beta} h_{\beta \mu}\right. \\
& \left.-\nabla_{\alpha} \nabla^{\nu} h_{\nu \mu}+\nabla_{\mu} \nabla_{\alpha}\left(H_{\rho}^{\rho}-\frac{2}{p-2} h_{\gamma}^{\gamma}\right)-\nabla_{\mu} \nabla^{\beta} h_{\beta \alpha}\right]
\end{aligned}
$$

which is sourced by

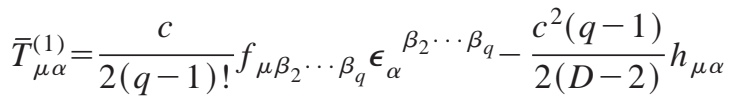

$$
\begin{aligned}
& =\frac{c}{2} \nabla_{\mu} \nabla_{\alpha} b+\frac{c}{2}\left(\square_{y} b_{\mu \alpha}-R_{\alpha}{ }^{\beta} b_{\mu \beta}\right)-\frac{c^{2}(q-1)}{2(D-2)} h_{\mu \alpha} .
\end{aligned}
$$

For $R_{\alpha \beta}$ we have

$$
\begin{aligned}
R_{\alpha \beta}^{(1)}= & -\frac{1}{2}\left[\left(\square_{x}+\square_{y}\right) h_{(\alpha \beta)}-2 R_{\alpha \gamma \delta \beta} h^{(\gamma \delta)}-R_{\alpha}^{\gamma} h_{(\gamma \beta)}\right. \\
& -R_{\beta}^{\gamma} h_{(\gamma \alpha)}+\frac{1}{q} g_{\alpha \beta}\left(\square_{x}+\square_{y}\right) h_{\gamma}^{\gamma}-\left(\frac{2}{q}+\frac{2}{p-2}\right) \\
& \left.\times \nabla_{\alpha} \nabla_{\beta} h_{\gamma}^{\gamma}+\nabla_{\alpha} \nabla_{\beta} H_{\mu}^{\mu}-\nabla_{\alpha} \nabla^{\mu} h_{\mu \beta}-\nabla_{\beta} \nabla^{\mu} h_{\mu \alpha}\right]
\end{aligned}
$$

while on the right-hand side, we find

$$
\begin{aligned}
\bar{T}_{\alpha \beta}^{(1)}= & \frac{c}{2(q-1) !}\left(f_{\alpha \gamma_{2} \cdots \gamma_{q}} \epsilon_{\beta}^{\gamma_{2} \cdots \gamma_{q}}+f_{\beta \gamma_{2} \cdots \gamma_{q}} \epsilon_{\alpha}{ }^{\gamma_{2} \cdots \gamma_{q}}\right) \\
& +\frac{c^{2}(q-1)}{2(q-1) !}\left(-h^{\gamma \delta}\right) \epsilon_{\alpha \gamma \theta_{3} \cdots \theta_{q}} \epsilon_{\beta \delta}{ }_{3} \cdots \theta_{q} \\
& -\frac{c^{2}(q-1)}{2(D-2)}\left(h_{(\alpha \beta)}+\frac{1}{q} g_{\alpha \beta} h_{\gamma}^{\gamma}\right)-\frac{c(q-1)}{(D-2)} g_{\alpha \beta}(f \cdot \epsilon) \\
& -\frac{q(q-1) c^{2}}{2(D-2) q !} g_{\alpha \beta}\left(-h^{\gamma \delta}\right) \epsilon_{\gamma \theta_{2} \cdots \theta_{q} \epsilon_{\delta}{ }^{\theta_{2} \cdots \theta_{q}}} \\
= & \frac{p-1}{D-2} g_{\alpha \beta} \square_{y} c b+\frac{q-1}{R^{2}} h_{(\alpha \beta)}-\frac{(q-1)^{2}}{q R^{2}} g_{\alpha \beta} h_{\gamma}^{\gamma},
\end{aligned}
$$


where we have used $(f \cdot \epsilon)=\square_{y} b \quad$ and $\quad f_{\alpha_{1} \cdots \alpha_{q}}$ $=(f \cdot \boldsymbol{\epsilon}) \boldsymbol{\epsilon}_{\alpha_{1} \cdots \alpha_{q}}=\boldsymbol{\epsilon}_{\alpha_{1} \cdots \alpha_{q}} \square_{y} b$.

We see that the modes of the graviton mix with the form modes $b$ and $b_{\mu}$. To solve the coupled systems, we must consider certain form equations as well. From the $\nabla^{M} F_{M \beta_{2}} \ldots \beta_{q}$ equation, ${ }^{4}$ we find the expression

$$
\begin{gathered}
\nabla^{M} f_{M \beta_{2} \cdots \beta_{q}}-c g^{\mu \nu} \Gamma_{\mu \nu}^{\gamma(1)} \epsilon_{\gamma \beta_{2} \cdots \beta_{q}}-c g^{\gamma \delta} \Gamma_{\gamma \delta}^{\theta(1)} \epsilon_{\theta \beta_{2} \cdots \beta_{q}} \\
-c(q-1) g^{\gamma \delta} \Gamma_{\gamma \beta_{2}}^{\theta(1)} \epsilon_{\delta \theta \beta_{3} \cdots \beta_{q}}=0,
\end{gathered}
$$

where we use the linearized Christoffel symbol,

$$
\Gamma_{M N}^{P(1)}=\frac{1}{2}\left(\nabla_{M} h_{N}^{P}+\nabla_{N} h_{M}^{P}-\nabla^{P} h_{M N}\right) .
$$

Contracting with the epsilon tensor on $\mathrm{M}_{q}$, Eq. (3.26) becomes

$$
\begin{gathered}
(q-1) !\left(\nabla_{\alpha}\left[\left(\square_{x}+\square_{y}\right) b+\frac{c}{2} H_{\mu}^{\mu}-\frac{c(p-1)}{p-2} h_{\gamma}^{\gamma}\right]\right. \\
\left.+\nabla^{\mu}\left[\square_{y} b_{\mu \alpha}-R_{\alpha}^{\beta} b_{\mu \beta}-c h_{\mu \alpha}\right]\right)=0
\end{gathered}
$$

Finally, from the $\nabla^{M} F_{M \mu \beta_{3} \ldots \beta_{q}}$ equation,

$$
\nabla^{M} f_{M \mu \beta_{3} \cdots \beta_{q}}-c g^{\gamma \alpha} \Gamma_{\gamma \mu}^{\delta(1)} \epsilon_{\alpha \delta \beta_{3} \cdots \beta_{q}}=0,
$$

which reduces to

$$
\begin{aligned}
& (q-2) !\left[\left(\square_{x}+\square_{y}-\frac{2(q-1)}{R^{2}}\right) \nabla_{[\alpha} b_{\beta] \mu}-\nabla^{\nu} \nabla_{\mu} \nabla_{[\alpha} b_{\beta] \nu}\right. \\
& \left.\quad-c \nabla_{[\alpha} B_{\beta] \mu}+2 R_{\alpha}^{\gamma}{ }_{\beta}^{\delta} \nabla_{[\gamma} b_{\delta] \mu}\right] \\
& \quad-(q-2) ! D_{\beta_{3}} D^{\nu} a_{\mu \nu \beta_{4} \cdots \beta_{q}} \epsilon_{\alpha \beta}^{\beta_{3} \cdots \beta_{q}} \\
& \quad+(q-2) !\left(\square_{x} \beta_{\mu}-\nabla^{\nu} \nabla_{\mu} \beta_{\nu}\right)=0 .
\end{aligned}
$$

We now expand these fields in spherical harmonics and collect like terms. Below we present the results, collecting related equations and indicating the origin of each expression as follows: (E1), (E2) and (E3) for the AdS, mixed and $\mathrm{M}_{q}$ Einstein equations, and (F1) and (F2) for the form equations (3.28) and (3.30), respectively.

Equations for the coupled scalars $\pi^{I}, b^{I}$, and $H^{I}$ :

$$
\text { (E3) } \begin{aligned}
& {\left[\left(\square_{x}+\square_{y}-\frac{2(q-1)^{2}}{R^{2}}\right) \pi^{I}\right.} \\
+ & \left.\square_{y}\left(H^{I}-\frac{2(D-2)}{q(p-2)} \pi^{I}\right)+\frac{2 q(p-1)}{(D-2)} \square_{y} c b^{I}\right] Y^{I}=0,
\end{aligned}
$$

\footnotetext{
${ }^{4}$ One may avoid explicit manipulation of Christoffel symbols by linearizing the equivalent equation $\partial_{M} \sqrt{-g} F^{M N_{2} \cdots N_{q}}=0$.
}

$$
\text { (E3) } \quad\left(H^{I}-\frac{2(D-2)}{q(p-2)} \pi^{I}\right) \nabla_{(\alpha} \nabla_{\beta)} Y^{I}=0,
$$

$$
\nabla_{\alpha}\left(\square_{x} b^{I}+\square_{y} b^{I}+\frac{c}{2} H^{I}-\frac{c(p-1)}{(p-2)} \pi^{I}\right) Y^{I}=0 .
$$

Equations for coupled vectors $b_{\mu}^{I}, B_{\mu}^{I}$ :

$$
\left(\operatorname{Max} B_{\mu}^{I}+\Delta_{y} B_{\mu}^{I}+\Delta_{y} c b_{\mu}^{I}-\frac{2(q-1)^{2}}{(p-1) R^{2}} b_{\mu}^{I}\right) Y_{\alpha}^{I}=0,
$$

$$
\text { (F2) } \nabla_{[\alpha}\left(\operatorname{Max} b_{\mu}^{I}+\Delta_{y} b_{\mu}^{I}-c B_{\mu}^{I}\right) Y_{\beta]}^{I}=0 \text {, }
$$

$$
\text { (F1) } \quad\left(\nabla^{\mu} b_{\mu}^{I} \Delta_{y}-c \nabla^{\mu} B_{\mu}^{I}\right) Y_{\alpha}^{I}=0 \text {, }
$$

$$
\text { (E3) } \quad\left(\nabla^{\mu} B_{\mu}^{I}\right) \nabla_{(\alpha} Y_{\beta)}^{I}=0 \text {, }
$$

where Max is the Maxwell operator. Equations for symmetric tensors $H_{\mu \nu}^{I}$ :

$$
\begin{aligned}
& \left(R_{\mu \nu}^{(1)}\left(H_{\rho \sigma}^{I}\right)-\frac{1}{2} \square_{y} H_{\mu \nu}^{I}+\frac{(q-1)^{2}}{(p-1) R^{2}} H_{\mu \nu}^{I}\right. \\
& +\frac{1}{2(p-2)} g_{\mu \nu}\left(\square_{x}+\square_{y}\right) \pi^{I}-\frac{(q-1)^{2}}{(p-2) R^{2}} g_{\mu \nu} \pi^{I} \\
& \left.+\frac{(q-1)}{(D-2)} g_{\mu \nu} \square_{y} c b^{I}\right) Y^{I}=0, \\
& \left(\begin{array}{c}
\left.-\nabla^{\nu} H_{\nu \mu}^{I}+\nabla_{\mu} H^{I}-\frac{(p+q-2)}{q(p-2)} \nabla_{\mu} \pi^{I}+\nabla_{\mu} c b^{I}\right) \nabla_{\alpha} Y^{I} \\
=0 .
\end{array}\right.
\end{aligned}
$$

Note that in Eq. (3.38), $R_{\mu \nu}^{(1)}$ is the linearized Ricci tensor for $\mathrm{AdS}_{p}$ only, evaluated on the field $H_{\rho \sigma}$. Finally, there remain a few decoupled equations:

$$
\begin{array}{r}
\text { (E3) }\left[\left(\square_{x}+\square_{y}\right) \delta_{\alpha}^{\gamma} \delta_{\beta}^{\delta}-2 R_{\alpha}^{\gamma \delta}{ }_{\beta}\right] \phi^{I} Y_{(\gamma \delta)}^{I}=0, \\
(\mathrm{~F} 2) \quad\left(\operatorname{Max} \beta_{\mu}^{h}\right) Y_{[\alpha \beta]}^{h}=0, \\
(\mathrm{~F} 2) \quad\left(\nabla^{\nu} b_{\nu \mu}^{I}\right) \nabla_{[\alpha} \nabla_{\beta]} Y^{I}=0 .
\end{array}
$$

Notice that in passing from Eq. (3.30) to Eq. (3.35), we commuted the $\square_{y}$ through the covariant derivative $\nabla_{\alpha}$, which not only produced precisely the Laplacian $\Delta_{y}$ acting on vectors, but also canceled all terms in Eq. (3.30) involving the Riemann tensor.

It is worth remarking that as a result, the properties of $\mathbf{M}_{q}$ enter into almost all these formulas only through the dimension $q$ and the radius $R$. Consequently we will be able to treat these equations in a completely unified way, and prove that for generic $\mathrm{AdS}_{p} \times \mathrm{M}_{q}$ backgrounds, all the corresponding modes satisfy the Breitenlohner-Freedman bound and cannot destabilize the background. The sole exception is the equation (3.40) for the scalars coming from graviton modes on the compact space, which explicitly involves the Riemann 
tensor on $\mathrm{M}_{q}$. There is thus no guarantee that the modes $\phi^{I}$ will possess the uniform stability properties for different choices of $\mathrm{M}_{q}$. Indeed, we will find that for $\mathrm{M}_{q}=\mathrm{S}^{q}$ these modes are harmlessly positive mass for all $q$, while for any product $\mathrm{M}_{q}=\mathrm{M}_{n} \times \mathrm{M}_{q-n}$ with $q<9$ they contain an instability.

\section{COUPLED SCALARS}

In this section, we consider the system of modes associated with the coupled scalars $\pi^{I}, b^{I}$, and $H^{I}$, Eqs. (3.31), (3.32), and (3.33).

For certain low-lying scalar spherical harmonics $Y^{I}$, some or all of their derivatives appearing in the equations of Sec. III B may vanish. Let us first treat the generic case where all derivatives of $Y^{I}$ in Eqs. (3.31), (3.32), and (3.33) are nonzero and hence the coefficients must vanish. Equation (3.32) then gives us a constraint which may be used to eliminate $H^{I}$ in favor of $\pi^{I}$. Substituting into Eq. (3.33), we find

$$
\left(\left(\square_{x}+\square_{y}\right) b^{I}-c \frac{(q-1)}{q} \pi^{I}\right) Y^{I}=0,
$$

while the second term in parentheses vanishes in Eq. (3.31). We obtain from Eqs. (3.31) and (4.1) the coupled system

$$
\begin{aligned}
L^{2} \square_{x}\left(\begin{array}{c}
b^{I} / c \\
\pi^{I}
\end{array}\right)= & (p-1)^{2}\left(\begin{array}{cc}
\frac{\lambda^{I}}{(q-1)^{2}} & \frac{R^{2}}{q(q-1)} \\
\frac{4 q \lambda^{I}}{(q-1) R^{2}} & \frac{\lambda^{I}}{(q-1)^{2}}+2
\end{array}\right) \\
& \times\left(\begin{array}{c}
b^{I} / c \\
\pi^{I}
\end{array}\right)
\end{aligned}
$$

where $\square_{y} Y^{I}=-\lambda^{I} Y^{I} / R^{2}$; that $\lambda^{I} \geqslant 0$ is straightforward and is shown in the Appendix. On diagonalizing this matrix we obtain the mass spectrum

$$
m^{2} L^{2}=\frac{(p-1)^{2}}{(q-1)^{2}}\left[\lambda+(q-1)\left(q-1 \pm \sqrt{4 \lambda+(q-1)^{2}}\right)\right] .
$$

We now wish to analyze the spectrum (4.3) to check stability. Extrema of Eq. (4.3) occur for

$$
1 \pm 2(q-1)\left(4 \lambda+(q-1)^{2}\right)^{-1 / 2}=0 .
$$

To satisfy Eq. (4.4) we must choose the negative sign, and we find a minimum at

$$
\lambda=\frac{3}{4}(q-1)^{2} .
$$

Substituting into Eq. (4.3), we find the elegant result that the minimum mass of the negative branch exactly saturates the Breitenlohner-Freedman bound independent of $p$ and $q$ :

$$
m_{\min }^{2} L^{2}=-\frac{1}{4}(p-1)^{2}=m_{B F}^{2} L^{2} .
$$

Since the positive branch leads to manifestly positive masses, we have proven there can be no unstable modes in this sector, at least for modes associated to generic spherical harmonics. We shall complete the proof by treating the special cases momentarily.

Although the spectrum (4.3) always saturates the BF bound as a smooth function of $\lambda$, there need not be physical states at the minimum, since only discrete values of $\lambda$ appear for given $\mathrm{M}_{q}$. If $\mathrm{M}_{q}=\mathrm{S}^{q}$, then the eigenvalues of the spherical harmonics are $\lambda=k(k+q-1)$, for integer $k \geqslant 0$, and the mass formulas for the two branches take on the form

$$
\begin{aligned}
& m_{-}^{2} L^{2}=\frac{(p-1)^{2}}{(q-1)^{2}} k(k-q+1), \\
& m_{+}^{2} L^{2}=\frac{(p-1)^{2}}{(q-1)^{2}}[k+2(q-1)](k+q-1) .
\end{aligned}
$$

The minimum (4.5) occurs for $\mathrm{S}^{q}$ at $k=(q-1) / 2$ in the minus branch. We notice that whenever $q$ is odd, there will be a mode with precisely the Breitenlohner-Freedman mass, while for $q$ even the lightest-mass states from this sector will appear just above the bound. This is consistent with what is already known about $\mathrm{AdS}_{4} \times \mathrm{S}^{7}$ and $\mathrm{AdS}_{7} \times \mathrm{S}^{4}[18,15,17]$.

Let us now examine the special cases. For $k=1$ on $S^{q}$, $\nabla_{(\alpha} \nabla_{\beta)} Y^{I}=0$ and we cannot use Eq. (3.32); this only occurs for maximally symmetric spaces, and hence is not a concern for other $\mathrm{M}_{q}$, where nonconstant $Y^{I}$ can be treated as above. Following [17] we may deal with this in one of two ways: either using a residual gauge invariance to impose the constraint anyway, or explicitly evaluating the remaining equations and showing that one linear combination drops out. We shall do the latter; for a discussion of the former, see [16].

We now consider Eq. (3.33) as a constraint to eliminate $H^{I}$ in favor of $\pi^{I}$ and $b^{I}$. The remaining equation (3.31) becomes

$$
\begin{aligned}
& \left(\square_{x}+\frac{3 q-2}{q} \square_{y}-\frac{2(q-1)^{2}}{R^{2}}\right) \pi^{I} \\
& \quad-\frac{R^{2}(p-1)}{(q-1)(D-2)}\left(\square_{x}+\square_{y}-\frac{2 q(q-1)}{R^{2}}\right) \\
& \quad \times \square_{y} c b^{I}=0 .
\end{aligned}
$$

In the case of the sphere, $\square_{y}=-q / R^{2}$ and we find an equation for a single mode,

$$
\left(\square_{x}-\frac{q(2 q-1)}{R^{2}}\right)\left(\pi^{I}+\frac{q(p-1)}{(q-1)(D-2)} c b^{I}\right)=0,
$$

which has the same mass as one would obtain from naively substituting $k=1$ into the positive branch of Eq. (4.7).

For constant $Y^{I}$ on any $\mathrm{M}_{q}$, all derivatives of $Y^{I}$ vanish and the only nontrivial equation is Eq. (3.31), which reduces to

$$
\left(\square_{x}-\frac{2(q-1)^{2}}{R^{2}}\right) \pi^{I}=0,
$$


where again the mass matches what one obtains by substituting $k=\lambda=0$ into the positive branch of Eq. (4.3). Thus we learn that a proper treatment extends the positive branch of Eq. (4.3) down to $k=0$, while the negative branch truncates at $k=2$ for $\mathrm{S}^{q}$ and $k=1$ for other $\mathrm{M}_{q}$.

The only remaining scalar fields associated to modes of the graviton are the $\phi^{I}$, which obey the uncoupled equation (3.40). These shall turn out to be the modes that can threaten stability. We shall return to these in Sec. VIII.

\section{COUPLED VECTORS}

We now consider the graviphoton $B_{\mu}$ and the form mode $b_{\mu}$ with which it mixes. We expect to find a massless vector for each Killing vector on $\mathbf{M}_{q}$ as well as a tower of massive fields, and indeed this is what we obtain. An additional $b^{2}\left(\mathrm{M}_{q}\right)$ massless vectors arise from the gauge potential, where $b^{2}\left(\mathrm{M}_{q}\right)$ is the second Betti number.

The relevant equations are Eqs. (3.34), (3.35), (3.36), and (3.37). One readily sees that Eq. (3.36) can be obtained from the divergence of Eq. (3.35). We obtain the following coupled system from Eqs. (3.34) and (3.35):

$$
\begin{aligned}
L^{2} \operatorname{Max}\left(\begin{array}{c}
c b_{\mu}^{I} \\
B_{\mu}^{I}
\end{array}\right)= & (p-1)^{2}\left(\begin{array}{cc}
\frac{\kappa^{I}}{(q-1)^{2}} & \frac{2(D-2)}{(p-1)(q-1)} \\
\frac{\kappa^{I}}{(q-1)^{2}} & \frac{\kappa^{I}}{(q-1)^{2}}+\frac{2}{p-1}
\end{array}\right) \\
& \times\left(\begin{array}{c}
c b_{\mu}^{I} \\
B_{\mu}^{I}
\end{array}\right)
\end{aligned}
$$

where $\Delta_{y} Y_{\alpha}^{I}=-\kappa^{I} Y_{\alpha}^{I} / R^{2}$. The masses that result are

$$
\begin{aligned}
m^{2} L^{2}= & \frac{(p-1)^{2}}{(q-1)^{2}} \kappa+(p-1) \\
& \times\left(1 \pm \sqrt{1+2 \frac{p-1}{(q-1)^{3}}(D-2) \kappa}\right)
\end{aligned}
$$

On a general Einstein space, we may derive the bound $\kappa^{I}$ $\geqslant 2(q-1)$, with equality when $Y_{\alpha}^{I}$ is a Killing vector, by considering $\int d^{q} y S_{\alpha \beta} S^{\alpha \beta} \geqslant 0$ with $S_{\alpha \beta} \equiv \nabla_{\alpha} Y_{\beta}+\nabla_{\beta} Y_{\alpha}$ (see the Appendix). For these Killing modes, the masses on the negative branch of Eq. (5.2) vanish. Hence we do indeed find a massless vector for each isometry of the compact space $\mathbf{M}_{q}$. For Killing modes (3.37) is trivially satisfied and does not constrain the vector fields.

The positive branch for $\kappa=2(q-1)$ yields a positive mass, and one can show that for both branches (5.2) monotonically increases with $\kappa$ for $\kappa \geqslant 2(q-1)$. Thus all vector modes are either massless or have positive mass. For the non-Killing modes (3.36) and (3.37) provide the usual divergence-free condition for massive vectors, while for the massive modes associated to the Killing vectors (3.36) accomplishes this by itself.

When the cohomology $H^{2}\left(\mathrm{M}_{q}\right)$ is nontrivial, harmonic 2-forms $Y_{[\alpha \beta]}^{h}$ give rise to $b^{2}\left(\mathrm{M}_{q}\right)$ additional massless vectors $\beta_{\mu}^{h}$, as we see from Eq. (3.41).

\section{GRAVITON AND TENSOR FIELDS}

We now establish the existence of the $p$-dimensional graviton and demonstrate the stability of the tower of massive symmetric two-index tensors. The graviton comes from the constant $Y^{I}$ mode of Eq. (3.38). Using Eq. (4.10), this reduces to

$$
R_{\mu \nu}^{(1)}\left(H_{\rho \sigma}^{I}\right)+\frac{p-1}{L^{2}} H_{\mu \nu}^{I}=0
$$

which is the correct fluctuation equation for a linearized graviton in $\mathrm{AdS}_{p}$.

For generic $Y^{I}$, the trace and longitudinal parts of Eq. (3.38) are redundant given Eqs. (3.31), (3.32), (3.33), and (3.39), which express the trace and divergence of $H_{\mu \nu}$ in terms of $\pi$ and $b$. One can use these equations to reduce Eq. (3.38) to

$$
\left[\left(\square_{x}+\square_{y}+\frac{2}{L^{2}}\right) H_{(\mu \nu)}^{I}-2 \nabla_{(\mu} \nabla_{\nu)} c b^{I}\right] Y^{I}=0
$$

A massive tensor field of mass $m^{2}$ is described by a field $\phi_{(\mu \nu)}$ which satisfies the wave equation and transversality constraints

$$
\begin{array}{r}
\left(\square_{x}-m^{2}\right) \varphi_{(\mu \nu)}=0, \\
\nabla^{\mu} \varphi_{(\mu \nu)}=0 .
\end{array}
$$

To bring Eq. (6.2) to this form, we follow [17]. Define $\phi_{(\mu \nu)}$ in terms of $H_{(\mu \nu)}$ by

$$
H_{(\mu \nu)}=\phi_{(\mu \nu)}+\nabla_{(\mu} \nabla_{\nu)}(u b+v \pi)
$$

where $u$ and $v$ are constants which can be determined by the following procedure, which we outline without full detail. The first step is to substitute Eq. (6.5) into Eq. (6.2) and require that $\phi_{(\mu \nu)}$ satisfy Eq. (6.3) with mass $m_{I}^{2}=\lambda^{I} / R^{2}$ $-2 / L^{2}$, where $-\lambda^{I} / R^{2}$ is as usual the eigenvalue of $\square_{y}$ on $Y^{I}$. The remaining terms are required to cancel which gives one condition to determine $u$ and $v$. The second condition is obtained by applying $\nabla^{\mu}$ to Eq. (6.5). The left side is expressed in terms of $b$ and $\pi$ using Eqs. (3.32) and (3.39), and one imposes Eq. (6.4). After commuting covariant derivatives, one finds two scalar conditions. Both contain the term $\square_{x}(u b+v \pi)$ which may be eliminated between them. The constants $u$ and $v$ may then be obtained by requiring that coefficients of the independent fields $b(x)$ and $\pi(x)$ vanish. The results are

$$
\begin{aligned}
& u=\frac{2 c(D-2)(p-2)}{(q-1) L^{2}\left(\frac{\lambda^{I}}{R^{2}}-\frac{p-2}{L^{2}}\right)} \\
& v=-\frac{D-2}{q(p-1)\left(\frac{\lambda^{I}}{R^{2}}-\frac{p-2}{L^{2}}\right)} .
\end{aligned}
$$


Strictly speaking the argument above does not apply to the $k=1$ graviton mode on $\mathrm{S}^{q}$ since it uses the constraint (3.32) which no longer follows from the Einstein equations. The simplest way to extend the argument is to use the unfixed conformal diffeomorphisms discussed in [17] to impose the constraint for $k=1$. The argument then goes through unchanged.

The apparent tensor mass $m_{I}^{2}$ is not positive for all geometries $\operatorname{AdS}_{p} \times \mathrm{M}_{q}$. However [17] one can examine $R_{\mu \nu}^{(1)}$ in Eq. (6.1) to see that the graviton itself has an apparent mass $-2 / L^{2}$. When this is subtracted one sees that higher tensor modes have positive mass $\lambda^{I} / R^{2}$. These modes transform in unitary representations of the $\mathrm{AdS}_{p}$ isometry group, and we have stability.

\section{UNCOUPLED FORM FLUCTUATIONS}

As we saw, the gauge potentials with zero and one indices on $\operatorname{AdS}_{p}$ mix with the graviton scalars and vectors. The remaining form fields are decoupled. It is easiest to treat them using a differential form notation. Thanks to the gauge condition (3.8), these may be written

$$
a(x, y)=\sum_{I} b^{I}(x) *_{q} d_{q} Y^{I}(y)+\sum_{h} \beta^{h}(x) Y^{h}(y) .
$$

The linearized equation of motion is simply

$$
d * d a=0 .
$$

Consider first the form $Y^{I}(y)$ with $n \geqslant 2$ indices on $\mathrm{M}_{q}$; the field $b^{I}$ then has $n$ indices on $\operatorname{AdS}_{p}$. Evaluating Eq. (7.2) and using the identities $*\left(A_{m}(x) B_{n}(y)\right)$ $=(-1)^{n(p-m)} *_{p}\left(A_{m}\right) *_{q}\left(B_{n}\right)$ and $d *_{q} Y^{I}=0$, we arrive at the equations

$$
\begin{aligned}
\left(d_{p} *_{p} d_{p} b^{I}\right) d_{q} Y^{I}+(-1)^{n^{2}}\left(*_{p} b^{I}\right) d_{q} \Delta_{y} Y^{I} & =0, \\
\left(d_{p} *_{p} b^{I}\right) \Delta_{y} Y^{I} & =0 .
\end{aligned}
$$

Equation (7.4) already appeared for the form with 2 indices on $\mathrm{AdS}_{p}$ as Eq. (3.42). It follows from Eq. (7.4) that Eq. (7.3) reduces to

$$
\left(\Delta_{x}-\frac{\kappa^{I}}{R^{2}}\right) b^{I}=0
$$

where $\Delta_{x}$ is the Laplacian on $\operatorname{AdS}_{p}$ and $\Delta_{y} Y^{I}=-\kappa^{I} Y^{I} / R^{2}$ is the eigenvalue of the Laplacian on $\mathrm{M}_{q}$, as before. Thus these are standard positive-mass modes resulting from the dimensional reduction.

The harmonic modes are even simpler; we find

$$
\left(d_{p}{ }_{p} \beta^{h}\right) Y^{h}=0 .
$$

Thus we have a massless form of appropriate rank for each cohomology class, as expected.

One potential modification of the action (2.1) is the addition of a Chern-Simons (CS) term

$$
\Delta S \sim \int A_{q-1} \wedge\left(F_{q}\right)^{n},
$$

where the wedge product is understood. Naturally, this is only possible when $q$ is even, and when an integer $n$ satisfying $n q=p+1$ can be found. (For $p=23, q=4$, one may add a CS term with $n=6$.) Notice that such a term breaks the duality symmetry between a theory with $F_{q}$, which we have used, and a dual $F_{p}$; results for the rest of this paper would be identical had we used $F_{p}$, but not in this instance. The modified action (7.7) leaves Einstein's equations unchanged, and modifies the form equation to

$$
d * F_{q}=\gamma\left(F_{q}\right)^{n},
$$

for some constant $\gamma$. In supersymmetric theories like 11dimensional supergravity, the constant $\gamma$ is fixed by supersymmetry. Absent supersymmetry or some other guiding principle, there is no preferred choice of $\gamma$. For $n \geqslant 2$ our solution (2.4), (2.7) is still valid since $F_{q} \wedge F_{q}$ vanishes. (For $n=1$, on the other hand, the Freund-Rubin background is not a solution.) Because $F_{q} \wedge F_{q}$ vanishes, Eq. (7.8) will begin to differ from Eq. (2.3) only at the $n-1$ order in perturbations. Hence, our linearized analysis will only be affected if $n=2$. Furthermore, for $f_{q} \wedge F_{q}$ to be nonzero, the fluctuation $f_{q}$ must be polarized entirely along $\operatorname{AdS}_{p}$. Hence, the addition of the term (7.7) can affect our analysis for only the single mode (3.14). We find the equation

$$
\left(\Delta_{x}+\Delta_{y}-2 c \gamma *_{p} d_{p}\right) b^{I} Y^{I}=0 .
$$

We notice that $\left(*_{p} d_{p}\right)^{2}=\Delta_{x}$ [for dimensions where Eq. (7.7) is possible]. We can thus factorize Eq. (7.9) into

$$
\begin{gathered}
\left(*_{p} d_{p}+m_{1}\right)\left(*_{p} d_{p}+m_{2}\right) b^{I} Y^{I}=0, \\
m_{1}+m_{2}=-2 c \gamma, \quad m_{1} m_{2}=-\kappa / R^{2},
\end{gathered}
$$

with the solution

$$
m_{1}=-c \gamma+\sqrt{c^{2} \gamma^{2}+\frac{\kappa}{R^{2}}}, \quad m_{2}=-c \gamma-\sqrt{c^{2} \gamma^{2}+\frac{\kappa}{R^{2}}} .
$$

There will be two towers, one annihilated by each of the factors in Eq. (7.10). The second-order equations are

$$
\left(\Delta_{x}-m_{i}^{2}\right) b^{I} Y^{I}=0,
$$

for $i=1,2$, and we see that $m_{i}^{2}$ are non-tachyonic masses regardless of $\gamma$.

\section{METRIC PERTURBATIONS ON $M_{q}$ AND STABILITY}

All the modes we have considered thus far have masses within the bounds for stability; moreover, we were able to show this for $\operatorname{AdS}_{p} \times \mathrm{M}_{q}$ where $\mathrm{M}_{q}$ is an arbitrary $q$-dimensional Einstein manifold. The only fields we have not considered as yet come from the traceless modes of the graviton on $\mathrm{M}_{q}$, and satisfy Eq. (3.40), which we repeat here: 


$$
\left[\left(\square_{x}+\square_{y}\right) \delta_{\alpha}^{\gamma} \delta_{\beta}^{\delta}-2 R_{\alpha}^{\gamma \delta}{ }_{\beta}\right] \phi^{I} Y_{(\gamma \delta)}^{I}=0 .
$$

It is possible to rewrite Eq. (8.1) in terms of the Lichnerowicz operator $\Delta_{L}$ and the Ricci tensor:

$$
\left[\square_{x}+\Delta_{L}+\frac{2(q-1)}{R^{2}}\right] \phi^{I} Y_{(\alpha \beta)}^{I}=0,
$$

but since $\Delta_{L}$ does not obey a universal inequality as $\square_{y}$ and $\Delta_{y}$ do, this form is not as useful. The presence of the Riemann tensor indicates that Eq. (8.1) can have different properties depending on the particular choice of $\mathrm{M}_{q}$. We give two examples, the sphere $\mathrm{M}_{q}=\mathrm{S}^{q}$ and a product space $\mathrm{M}_{q}$ $=\mathrm{M}_{n} \times \mathrm{M}_{q-n}$, and show that the former contains only stable modes while the latter possesses an instability for $q<9$.

For $\mathrm{M}_{q}=\mathrm{S}^{q}$, the Riemann tensor has the maximally symmetric form $R_{\alpha \beta \gamma \delta}=\left(g_{\alpha \gamma} g_{\beta \delta}-g_{\alpha \delta} g_{\beta \gamma}\right) / R^{2}$. Equation (8.1) reduces to

$$
\left[\left(\square_{x}+\square_{y}\right)-\frac{2}{R^{2}}\right] \phi^{I} Y_{(\alpha \beta)}^{I}=0 .
$$

All these modes are manifestly positive-mass. We thus complete our demonstration of the stability of the $\operatorname{AdS}_{p} \times S^{q}$ background for all $p$ and $q$.

In [8] and [9] it was pointed out that $\mathrm{AdS}_{4} \times \mathrm{M}_{n} \times \mathrm{M}_{7-n}$ and $\mathrm{AdS}_{7} \times \mathrm{S}^{2} \times \mathrm{S}^{2}$, respectively, were unstable to a perturbation in which one compact space becomes uniformly larger and the other smaller keeping the total volume fixed. We now generalize this to an arbitrary product of Einstein spaces $\mathbf{M}_{q}=\mathbf{M}_{n} \times \mathrm{M}_{q-n}$ with $n \geqslant 2$. Let $a, b$ denote indices on $\mathbf{M}_{n}$ and $i, j$ denote indices on $\mathbf{M}_{q-n}$. If the radii of the spaces are $R_{1}$ and $R_{2}$, requiring that the total compact space is also Einstein imposes the relation

$$
\frac{n-1}{R_{1}^{2}}=\frac{q-n-1}{R_{2}^{2}}=\frac{q-1}{R^{2}} .
$$

Consider now the mode

$$
h_{a b}=\frac{1}{n} g_{a b} \phi(x), \quad h_{i j}=-\frac{1}{q-n} g_{i j} \phi(x),
$$

which satisfies $h_{\alpha}^{\alpha}=0$ as well as the gauge condition (3.5) and therefore obeys Eq. (8.1). This perturbation increases the radius of one of the Einstein spaces and decreases the radius of the other keeping the total volume constant (to first order). Evaluating Eq. (8.1), we find

$$
\left[\square_{x}+\frac{2(q-1)}{R^{2}}\right] \phi^{I}=0 .
$$

Thus this mode has the mass

$$
m^{2} L^{2}=-\frac{2(p-1)^{2}}{(q-1)}=\frac{8}{q-1} m_{B F}^{2} L^{2} .
$$

Consequently the Breitenlohner-Freedman bound (3.1) is violated for $q<9$. This result is independent of $p$, and de- pends on the internal space only in that it is a product of Einstein spaces that is itself Einstein with total dimension $q$; in particular the relative dimension of the spaces is irrelevant.

One may wonder about other fluctuations obeying Eq. (8.1), and whether they may place even more stringent constraints on the requirements for stability. The field (8.5) is the lowest in a tower of modes that are traces on each individual space in the product, but traceless overall. Higher excitations will have more positive masses from the $\square_{y}$ term. The remaining modes are traceless on each $\mathrm{M}_{n}$ and $\mathrm{M}_{q-n}$, namely $h_{(a b)}, h_{(i j)}$, and $h_{a i}$. For $h_{a i}$ we find the universal result

$$
\left(\square_{x}+\square_{y}\right) h_{a i}=0,
$$

which is obviously stable, while for either of the other two we have effectively a copy of Eq. (8.1) but involving the Riemann tensor of just one of the spaces in the product

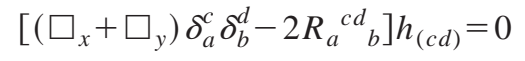

and similar for $h_{(i j)}$. This obviously depends on the details of $\mathrm{M}_{n}$. One observation we can make is that if $\mathrm{M}_{n}$ itself is a product (and so the original compact space $\mathrm{M}_{q}$ is a product of three or more manifolds), a mode analogous to Eq. (8.5) will have a mass $m^{2}=2(n-1) / R_{1}^{2}=2(q-1) / R^{2}$, where the last equality comes from Eq. (8.4), and thus will be unstable precisely when Eq. (8.5) is; and hence no new instability automatically arises for products of three or more spaces beyond that already generically present for a product of two.

\section{AdS $_{4}$ VACUA OF MASSIVE TYPE IIA}

Massive type IIA supergravity has $\mathrm{AdS}_{4} \times \mathrm{M}_{6}$ vacua [13] which are non-supersymmetric and whose stability, to our knowledge, has never been investigated. ${ }^{5}$ Even the existence of these solutions is non-trivial, since there is a potential term for the dilaton which pushes it toward weak coupling. What makes $\mathrm{AdS}_{4} \times \mathrm{M}_{6}$ vacua possible is that a uniform $\mathrm{RR}$ field strength, $F_{4}$ or $F_{6}$ according to taste, pushes the dilaton toward strong coupling, and there is an extremum of this total potential where the dilaton can be constant.

The extremum is in fact a maximum, but it does not make sense to ask whether second derivative of the total dilaton potential alone satisfies the $\mathrm{BF}$ bound, because the dilaton couples non-trivially to the form and to the graviton. This mixing means that the coupled scalars sector requires a more intricate analysis than before. The result will be that the apparent $s$-wave tachyon coming from a naive analysis of the dilaton potential is completely erased (effectively, it is a gauge artifact), but for $S^{6}$ there is a $d$-wave and an $f$-wave mode which violates the BF bound, rendering this vacuum

\footnotetext{
${ }^{5}$ There is also a supersymmetric (and necessarily stable) vacuum which is a fibration of $\mathrm{AdS}_{6}$ over $\mathrm{S}^{4}$ with a non-trivial dilaton. It is the near-horizon geometry of the D4-D8 system [23]. It would be interesting to explore the properties of this background as well as generalizations of it where $S^{4}$ is replaced by other manifolds, but we will not do so here.
} 
unstable! To our knowledge, this is the first time that a product of AdS and a round sphere is unstable. We also show that for $\mathrm{M}_{6}=\mathrm{S}^{n} \times \mathrm{S}^{6-n}$ the $\mathrm{BF}$ bound is violated within the coupled scalar sector, as well as having the same purely gravitational instability found earlier, where one factor shrinks while the other grows.

The remaining modes, outside the coupled scalar sector, satisfy the same equations as in the generic $\operatorname{AdS}_{p} \times \mathrm{M}_{q}$ systems we already considered. Thus the traceless graviton on $\mathrm{M}_{6}$ joins the coupled scalars as a possible source of instability. We do not analyze other Einstein manifolds $\mathrm{M}_{6}$ explicitly, but we provide the tools needed for such an analysis. It is still possible that there exist stable $\operatorname{AdS}_{4} \times \mathrm{M}_{6}$ vacua.

To make the discussion similar to our previous analysis, let us express the action for massive type IIA in terms of a six-form field strength, which is essentially the Hodge dual of the usual four-form:

$$
\begin{aligned}
S= & \frac{1}{2 \kappa^{2}} \int d^{10} x \sqrt{g}\left[R-\frac{1}{2}(\partial \phi)^{2}-\frac{1}{2} \xi^{2} F_{6}^{2}\right. \\
& \left.-\frac{m^{2}}{8} \xi^{-10}\right] \quad \text { where } \quad \xi=e^{-\phi / 4},
\end{aligned}
$$

and we include a $1 / 6$ ! in the definition of $F_{6}^{2}$, as in [24]. We also include a factor of $1 / q$ ! in the inner product of forms, $\omega_{q} \cdot \tilde{\omega}_{q}$. The equations of motion are

$$
\begin{aligned}
& R_{M N}= \frac{m^{2}}{64} \xi^{-10} g_{M N} \\
&+\frac{1}{2} \partial_{M} \phi \partial_{N} \phi+\frac{\xi^{2}}{2 \times 5 !} \\
& \times F_{M P_{1} P_{2} P_{3} P_{4} P_{5} F_{N} P_{1} P_{2} P_{3} P_{4} P_{5}} \\
&-\frac{5}{16} \xi^{2} g_{M N} F_{6}^{2}, \\
& \square-\frac{5}{16} m^{2} \xi^{-10}+\frac{\xi^{2}}{4} F_{6}^{2}=0, \\
& d * \xi^{2} F_{6}=0,
\end{aligned}
$$

and there is an $\mathrm{AdS}_{4} \times \mathrm{M}_{6}$ background with $\phi=0, F_{6}$ $=c \mathrm{vol}_{\mathrm{M}_{6}}$. We readily derive the relations

$$
c^{2}=F_{6}^{2}=\frac{5}{4} m^{2}=\frac{10}{L^{2}}=\frac{25}{R^{2}},
$$

where $L$ is the radius of curvature of $\mathrm{AdS}_{4}$, such that $R_{\mu \nu}$ $=-\left(3 / L^{2}\right) g_{\mu \nu}$, and $R$ is the radius of curvature of $\mathrm{M}_{6}$, such that $R_{\alpha \beta}=\left(5 / R^{2}\right) g_{\alpha \beta}$.

Just as for $\operatorname{AdS}_{p} \times \mathrm{M}_{q}$, we wish to linearize around the background to obtain the mass spectrum. For the coupled scalar sector, we wish to focus on perturbations of the form $g_{M N} \rightarrow g_{M N}+h_{M N}$ with $h_{\mu \nu}=\frac{1}{4} g_{\mu \nu} h_{\lambda}^{\lambda}$ and $h_{\alpha \beta}=\frac{1}{6} g_{\alpha \beta} h_{\gamma}^{\gamma}$. Also let $\delta \phi$ be the perturbation in $\phi$ and let $f_{6}$ be the perturbation in $F_{6}$, where, as before, we write

$$
h_{\alpha}^{\alpha}=\pi, \quad f_{6}=d a_{5}, \quad \text { where } \quad a_{5}=*_{6} d b .
$$

The algebraic relation $h_{\mu}^{\mu}+h_{\alpha}^{\alpha}=\frac{1}{3} h_{\alpha}^{\alpha}$ follows from the symmetric traceless part of the Einstein equations, as before. It is now possible to derive coupled second order equations relating $\delta \phi, b$, and $\pi$ from the variations of the $R_{\alpha}^{\alpha}$ Einstein equation, the scalar equation, and the form equation, using the algebraic relation when needed to eliminate $h_{\mu}^{\mu}$ in favor of $h_{\alpha}^{\alpha}$. We use a form notation in this section for convenience.

The $R_{\alpha}^{\alpha}$ equation is

$$
R_{\alpha}^{\alpha}=\frac{3}{4 L^{2}} \xi^{-10}+\frac{9}{8} \xi^{2} F_{6}^{2}+\frac{1}{2} \partial^{\alpha} \phi \partial_{\alpha} \phi .
$$

Using Eqs. (3.16), (9.3), and (9.4), we find

$$
\begin{aligned}
\delta R_{\alpha}^{\alpha} & =-\frac{2}{L^{2}} h_{\alpha}^{\alpha}-\frac{1}{2}\left(\square_{x}+\square_{y}\right) h_{\alpha}^{\alpha}-\frac{1}{2} \square_{y}\left(h_{\mu}^{\mu}+h_{\alpha}^{\alpha}\right)+\frac{1}{6} \square_{y} h_{\alpha}^{\alpha} \\
& =-\frac{15}{4 L^{2}} \delta \phi+\frac{9}{4} c \square_{y} b-\frac{45}{4 L^{2}} h_{\alpha}^{\alpha},
\end{aligned}
$$

where we have used the fact that $\square_{y}=*_{6} d *_{6} d$ acting on $b$. The algebraic relation allows us to simplify this to

$$
\left(\square_{x}+\square_{y}\right) \pi-\frac{37}{2 L^{2}} \pi-\frac{15}{2 L^{2}} \delta \phi+\frac{9}{2} c \square_{y} b=0 .
$$

For the scalar, the equation of motion is

$$
\square \phi-\frac{5}{2 L^{2}} \xi^{-10}+\frac{\xi^{2}}{4} F_{6}^{2}=0 .
$$

Linear variation around the background gives

$$
\begin{gathered}
\left(\square_{x}+\square_{y}\right) \delta \phi-\frac{25}{4 L^{2}} \delta \phi-\frac{1}{8} \delta \phi F_{6}^{2}+\frac{1}{2} F_{6} \cdot f_{6} \\
-\frac{1}{4} h^{\alpha \beta} F_{\alpha \gamma_{1} \ldots \gamma_{5}} F_{\beta}^{\gamma_{1} \ldots \gamma_{5}} \frac{1}{5 !}=0,
\end{gathered}
$$

which upon simplification and use of $F_{6} \cdot f_{6}=c \square_{y} b$ becomes

$$
\left(\square_{x}+\square_{y}\right) \delta \phi-\frac{15}{2 L^{2}} \delta \phi+\frac{1}{2} c \square_{y} b-\frac{5}{2 L^{2}} \pi=0 .
$$

The variation of the form equation is

$$
d(\delta *) F_{6}-d * \frac{1}{2} \delta \phi F_{6}+d * f_{6}=0,
$$

where $\delta *$ indicates the variation in the Hodge dual. After some algebra this becomes

$$
\frac{c}{2} d\left(h_{\mu}^{\mu}-h_{\alpha}^{\alpha}-\delta \phi\right) \wedge \operatorname{vol}_{4}+d\left(\square_{x}+\square_{y}\right) b \wedge \operatorname{vol}_{4}=0,
$$

and so, using the algebraic relation, we obtain

$$
\left(\square_{x}+\square_{y}\right) b-\frac{5 c}{6} \pi-\frac{c}{2} \delta \phi=0 .
$$


Gathering everything together, setting $b=c L^{2} B$ for convenience, and recalling that $c^{2}=10 / L^{2}$, one obtains the following system of equations:

$$
\begin{array}{r}
\left(\square_{x}+\square_{y}\right) B-\frac{5}{6 L^{2}} \pi-\frac{1}{2 L^{2}} \delta \phi=0 \\
\left(\square_{x}+\square_{y}\right) \pi-\frac{37}{2 L^{2}} \pi-\frac{15}{2 L^{2}} \delta \phi+45 \square_{y} B=0 \\
\left(\square_{x}+\square_{y}\right) \delta \phi-\frac{5}{2 L^{2}} \pi-\frac{15}{2 L^{2}} \delta \phi+5 \square_{y} B=0 .
\end{array}
$$

This results in

$$
L^{2} \square_{x}\left(\begin{array}{c}
B \\
\pi \\
\delta \phi
\end{array}\right)=\left(\begin{array}{ccc}
\frac{2}{5} \lambda & \frac{5}{6} & \frac{1}{2} \\
18 \lambda & \frac{2}{5} \lambda+\frac{37}{2} & \frac{15}{2} \\
2 \lambda & \frac{5}{2} & \frac{2}{5} \lambda+\frac{15}{2}
\end{array}\right)\left(\begin{array}{c}
B \\
\pi \\
\delta \phi
\end{array}\right),
$$

where as before $-R^{2} \square_{y} Y^{I}=\lambda Y^{I}$. We find the mass eigenvalues

$$
\begin{gathered}
m^{2} L^{2}=\frac{2}{5} \lambda+6, \quad \frac{2}{5} \lambda+10+2 \sqrt{25+4 \lambda}, \\
\text { and } \frac{2}{5} \lambda+10-2 \sqrt{25+4 \lambda} .
\end{gathered}
$$

The Breitenlohner-Freedman bound for $p=4$ is $m^{2} L^{2} \geqslant$ $-9 / 4$. We see that the first two towers in Eq. (9.16) are harmless (in fact they are not even tachyonic), but the third tower will violate the BF bound if some value of $\lambda$ falls in the interval

$$
\lambda_{\text {unstable }} \in\left(\frac{155}{8}-5 \sqrt{\frac{5}{2}}, \frac{155}{8}+5 \sqrt{\frac{5}{2}}\right) \approx(11.47,27.28) .
$$

For $S^{6}$, we have $\lambda=k(k+5)$, for which $k=2,3$ gives values in the interval (9.17). Thus for both $d$ - and $f$-waves, the eigen-combinations of $B, \pi$, and $\delta \phi$ corresponding to the third eigenvalue in Eq. (9.16) are unstable modes of the $\mathrm{AdS}_{4} \times \mathrm{S}^{6}$ solution. They have the common mass $m^{2} L^{2}=$ $-12 / 5$.

It is interesting that in fact all values of $m^{2} L^{2}$ that occur for $\mathrm{AdS}_{4} \times \mathrm{S}^{6}$ in the coupled scalar sector are rational: upon substituting $\lambda=k(k+5)$ into Eq. (9.16), we obtain

$$
m^{2} L^{2}=\frac{2 k^{2}}{5}+2 k+6, \quad \frac{2 k^{2}}{5}+6 k+20, \quad \text { and } \quad \frac{2 k^{2}}{5}-2 k .
$$

However the corresponding dimensions of operators in a hypothetical three-dimensional CFT are not rational.
Instabilities can occur in the coupled scalar sector of other $\mathrm{M}_{q}$ as well. As an example, consider $\mathrm{M}_{6}=\mathrm{S}^{n} \times \mathrm{S}^{6-n}$. For product spherical harmonics on the two spheres labeled by $\left(k_{1}, k_{2}\right)$, we find several unstable modes in the interval (9.17): $(1,1),(0,2)$, and $(1,2)$ for $n=2$, and $(1,1),(2,0)$, and $(0,2)$ for $n=3$.

As in Sec. IV, the constraint relating $h_{\mu}^{\mu}$ and $h_{\alpha}^{\alpha}$ no longer obtains for the $k=1$ case on $S^{6}$, so a more careful analysis must be performed. Without imposing the algebraic constraint, the dilaton equation (9.10) is unmodified, while Eqs. (9.6) and (9.12) become

$$
\left(\square_{x}+\square_{y}\right) \pi+\square_{y}(H+\pi)-\frac{1}{3} \square_{y} \pi-\frac{37}{2 L^{2}} \pi-\frac{15}{2 L^{2}} \delta \phi
$$

$$
\begin{aligned}
+ & \frac{9}{2} c \square_{y} b=0, \\
& \left(\square_{x}+\square_{y}\right) b-\frac{c}{2} \pi+\frac{c}{2} H-\frac{c}{2} \delta \phi=0 .
\end{aligned}
$$

For $k=1$, we have $\square_{y}=-12 / 5 L^{2}$. The dilaton equation (9.10) then becomes

$$
\begin{array}{r}
\left(\square_{x}-\frac{99}{10 L^{2}}\right) \delta \phi-\frac{5}{2 L^{2}} \pi-\frac{6}{5 L^{2}} c b \\
=\left(\square_{x}-\frac{99}{10 L^{2}}\right) \delta \phi-\frac{5}{2 L^{2}} \sigma=0,
\end{array}
$$

which defines $\sigma \equiv \pi+\frac{12}{25} c b$. Next, using Eq. (9.20) we can show that

$$
\square_{x} \pi+\square_{y} H=\square_{x} \sigma-\frac{12}{5 L^{2}} \sigma-\frac{12}{5 L^{2}} \delta \phi,
$$

which allows us to write Eq. (9.19) as

$$
\square_{x} \sigma-\frac{249}{10 L^{2}} \sigma-\frac{99}{10 L^{2}} \delta \phi=0 .
$$

As in the examples without a coupled dilaton, one linear combination of fields has dropped out of the $k=1$ system. We can now diagonalize Eqs. (9.21) and (9.23). We discover the mass eigenvalues

$$
m^{2} L^{2}=\frac{42}{5}, \quad m^{2} L^{2}=\frac{132}{5},
$$

which coincide with the $k=1$ masses in the first two towers of Eq. (9.18).

The constant $Y^{I}$ sector is straightforward for all $\mathrm{M}_{6}$. The form equation no longer obtains, and the $b$ mode does not exist, leaving only the equations

$$
\square_{x} \pi=\frac{37}{2 L^{2}} \pi+\frac{15}{2 L^{2}} \delta \phi,
$$




$$
\square_{x} \delta \phi=\frac{15}{2 L^{2}} \delta \phi+\frac{5}{2 L^{2}} \pi,
$$

with corresponding positive-mass eigenvalues

$$
m^{2} L^{2}=6, \quad m^{2} L^{2}=20 .
$$

These are exactly the masses obtained from the first two towers in Eq. (9.16) with $k=\lambda=0$. Thus the general "rule of thumb" (valid in all cases we have considered, as well as in the familiar supersymmetric examples) is that one simply drops the most tachyonic mode from the first two partial waves in the coupled scalar sector.

It is not hard to see that the remaining equations of motion are basically unmodified from the analysis of previous sections. The dilaton fluctuation $\delta \phi$ cannot appear in the other polarizations of the form equation, where the background field strength vanishes. Hence these are unchanged from before. In the Einstein equations, it is straightforward that $\delta \phi$ does not appear in the $R_{\mu \alpha}$ equation or in parts of the $R_{\alpha \beta}$ equation other than those treated already by considering the trace. Owing to the relations (9.3) arising from the requirement that the compact space is Einstein, these equations are identical to those we already studied once written in terms of $L$. The dilaton fluctuation and the other scalars do appear in the $R_{\mu \nu}$ equation, analogous to the appearance of $\pi, b$, and $H$ in Eq. (3.38), but this leads only to a scalar expression linearly dependent on the ones we have considered earlier.

Consequently, we can employ the work we have already done wholesale. In particular, we again have the potential source of instability from the set of scalars $\phi^{I}$, obeying Eq. (8.1). Hence we learn that general product spaces are again unstable against having one factor shrink while the other grows.

\section{POSSIBLE CFT DUALS}

As discussed in the Introduction, this investigation was motivated by the proposal [11] that the case $D=p+q=27$ with a 4-form field is the low-energy limit of a "bosonic $\mathrm{M}$ theory," and that its $\mathrm{AdS}_{4} \times \mathrm{S}^{23}$ compactification has a $\mathrm{CFT}_{3}$ dual in the framework of the AdS/CFT correspondence. Since an $\mathrm{AdS}_{p} \times \mathrm{S}^{q}$ compactification has been shown to be stable, it is interesting to speculate in general about possible $\mathrm{CFT}_{d}$ duals (with $d=p-1$ ). We give a very heuristic discussion which emphasizes the pattern of operator dimensions.

For scalar operators the basic AdS/CFT relation $\Delta(\Delta$ $-d)=m^{2} L^{2}$ admits the two roots

$$
\Delta_{ \pm}=\frac{d}{2} \pm \frac{1}{2} \sqrt{d^{2}+4 m^{2} L^{2}}
$$

If the mass satisfies the inequality $m^{2} L^{2} \geqslant-d^{2} / 4+1$, then only the assignment $\Delta_{+}$obeys the unitarity bound $\Delta \geqslant d / 2$ -1 . (This bound is saturated for a free massless scalar field in $d$ dimensions.) But for $-d^{2} / 4 \leqslant m^{2} L^{2} \leqslant-d^{2} / 4+1$, both $\Delta_{+}$and $\Delta_{-}$are, a priori, consistent choices for the scale dimension of the dual operator. On general grounds it seems most natural to choose the larger of the two dimensions, $\Delta_{+}$, as the dimension of the operator, because only then can one compute correlators by straightforwardly imposing a boundary condition on the larger of the two linearly independent solutions of the scalar. If $\Delta_{-}$is chosen as the dimension, then to obtain field theory correlators one must make a Legendre transform of the $\Delta_{+}$results. These points were discussed in [25], where also a particular example was exhibited where the $\Delta_{-}$dimension was needed. In this example, the field theory was supersymmetric, and the operator was a chiral primary, so its anomalous dimension could be worked out purely on field theory grounds as the sum of the anomalous dimensions of its factors. The computation is rigorous because all the dimensions are dictated by a $U(1)_{R}$ current which is obviously additive.

The mass eigenvalues of coupled scalars of general $\operatorname{AdS}_{p} \times \mathrm{S}^{q}$ compactifications are given in Eq. (4.7). Since $m_{+}^{2}>0$, the operator duals of positive branch scalars have the unique dimension assignments

$$
\Delta=\frac{p-1}{2}\left[1+\frac{2}{q-1}\left(k+\frac{3(q-1)}{2}\right)\right] .
$$

For the negative branch of the scalar mass spectrum, there are the two possibilities

$$
\Delta_{ \pm}=\frac{p-1}{2}\left[1 \pm \frac{2}{q-1}\left|k-\frac{q-1}{2}\right|\right] .
$$

In accord with the discussion in the previous paragraph the negative root is a possible choice in the range

$$
\left|k-\frac{q-1}{2}\right| \leqslant \frac{q-1}{d} .
$$

Recall that $k$ indicates the $S O(q+1)$ representation formed from $k$ factors of the vector, then symmetrized with the trace removed.

For the purposes of orientation, let us recall a familiar result for $\mathrm{AdS}_{5} \times \mathrm{S}^{5}$. Here the chiral primary operators are $\operatorname{tr} X^{\left(I_{1} \ldots X^{\left.I_{k}\right)}\right.}$ in $\mathcal{N}=4$ super-Yang-Mills theory, where $\left(I_{1} \ldots I_{k}\right)$ indicates the symmetric traceless combination. Their AdS duals are the coupled fluctuations of the metric and the five-form on the negative branch that leads to Eq. (10.3). The dimensions are $\Delta(k)=k=2,3,4,5, \ldots$, and one always chooses $\Delta_{+}$. The anomalous dimensions vanish: $\Delta(k)=k$ is the free-field result. A similar story holds for $\mathrm{AdS}_{4} \times \mathrm{S}^{7}$, with $\Delta(k)=k / 2$, except that one must choose $\Delta_{-}$ for $k=2$. Some of these operators are thought of as coming from $\operatorname{tr} X^{\left(I_{1}\right.} \ldots X^{\left.I_{k}\right)}$ on coincident D2-branes, and for the others one must dualize the vector boson into an eighth scalar. Free field counting still applies, and it can be backed up by a supersymmetry argument as for the $\mathrm{AdS}_{5} \times \mathrm{S}^{5}$ case. Lastly, for $\mathrm{AdS}_{7} \times \mathrm{S}^{4}$, the dimensions are $\Delta(k)=2 k$, and one always chooses $\Delta_{+}$. A free field understanding is lacking in this mysterious $(2,0)$ theory, but as before a link can be established between the $R$ symmetry and the dimension which guarantees that $\Delta(k)$ is linear in $k$.

Let us begin the discussion of the spectra for general $p$ and $q$ by observing that it is doubly remarkable that both the quadratic equation for scalar masses and the equation $\Delta(\Delta$ 
$-d)=m^{2} L^{2}$ have rational roots in the general case. This is an aesthetically pleasing point for a putative CFT dual, but unfortunately it is the end of the good news.

Focusing on the negative branch (10.3) makes sense, since these were the simplest operators in cases which we understand. Starting with our free field prejudices, we might suspect that the $k$ 'th operator would be expressible as $\operatorname{tr} X^{\left(I_{1}\right.} \cdots X^{\left.I_{k}\right)}$, and that its dimension $\Delta(k)$ is linear in $k$. Then we arrive at $\Delta(k)=[(p-1) /(q-1)] k$. For example, $\Delta(k)=\frac{3}{22} k$ for $\mathrm{AdS}_{4} \times \mathrm{S}^{23}$. This does not make sense because $k=2$ gives $\Delta=\frac{3}{11}<\frac{1}{2}$, the free scalar dimension. That is, we tried to choose $\Delta_{-}$in a range where only $\Delta_{+}$was possible. The general result is that a linear spectrum of dimensions $\Delta(k)$ is permitted provided

$$
q-1 \leqslant \frac{4(p-1)}{p-3}
$$

If this inequality fails, as in the case $\mathrm{AdS}_{4} \times \mathrm{S}^{23}$, then some operators of low $S O(q+1)$ charge will have a larger dimension than operators of higher $S O(q+1)$ charge, which we may view as a failure of the free-field intuition that singlet operators are built from fundamental fields whose dimensions add. It does not mean, however, that there cannot be a CFT dual: for instance, it is consistent with the unitarity bound to choose $\Delta_{+}$uniformly, which produces a spectrum $\Delta(k)$ with a kink about $k=(q-1) / 2$. More arcane choices may also be imagined. In the absence of supersymmetry or some input from field theory, we have no way of deciding between the alternatives.

Let us now discuss the spectra of coupled vectors for general $\operatorname{AdS}_{p} \times \mathrm{S}^{q}$ compactifications. Inserting the eigenvalue formula $\kappa=(k+1)(k+q-2)$ for vector spherical harmonics in Eq. (5.2), we find the masses

$$
m^{2} L^{2}=\frac{(p-1)^{2}}{(q-1)^{2}}(k+1)(k+q-2)+(p-1)\left(1 \pm \sqrt{1+2 \frac{p-1}{(q-1)^{3}}(p+q-2)(k+1)(k+q-2)}\right) .
$$

These mass eigenvalues are generically irrational (although they are rational for the supersymmetric compactifications $\mathrm{AdS}_{4} \times \mathrm{S}^{7}, \mathrm{AdS}_{7} \times \mathrm{S}^{4}$, and $\left.\mathrm{AdS}_{5} \times \mathrm{S}^{5}\right)$. Irrationality persists for vector scale dimensions (except for Killing vectors, where $\left.m^{2}=0\right)$ :

$$
\Delta=\frac{1}{2}\left[d+\sqrt{(d-2)^{2}+4 m^{2}}\right] .
$$

In particular, $\mathrm{AdS}_{4} \times \mathrm{S}^{23}$ has irrational masses and dimensions for massive vectors.

It is certainly remarkable that the scalars dual to chiral primary operators in the well-understood $\mathrm{AdS}_{5} \times \mathrm{S}^{5}$, $\mathrm{AdS}_{4} \times \mathrm{S}^{7}$, and $\mathrm{AdS}_{7} \times \mathrm{S}^{4}$ vacua still lead to rational dimensions for general $p$ and $q$. If Eq. (10.5) is violated and a linear spectrum of dimensions is impossible for scalars, then it seems difficult to imagine a concise understanding based on a Lagrangian. The fact that massive vector modes generically have irrational dimensions also makes it seem less likely that a purely field theoretic formulation of the putative dual CFT will be accessible in the near future.

The $\mathrm{AdS}_{4} \times \mathrm{S}^{6}$ compactification presents an even less rosy picture, in that the BF bound is violated. Obvious candidates for a brane realization of this vacua (involving D2-branes and D8-branes) seem also to be unstable, only the instability is usually in the form of a tadpole instead of a tachyon. It would be very interesting if a stable $\mathrm{AdS}_{4} \times \mathrm{M}_{6}$ vacuum could be found for appropriate $\mathrm{M}_{6}$, corresponding to some analyzable type I' brane configuration. It would also be satisfying if one could start with some unstable D2-D8 construction and show that in an appropriate near-horizon limit the brane instability reduces to the violations of the $\mathrm{BF}$ bound that we have observed. ${ }^{6}$

Finally, let us extend some remarks on thermodynamics made in [11] for the $\mathrm{AdS}_{4} \times \mathrm{S}^{23}$ and $\mathrm{AdS}_{23} \times \mathrm{S}^{4}$ cases. An obvious measure of the number of degrees of freedom in a CFT in $p-1$ dimensions is the ratio $c_{\text {thermo }}=S /\left(V T^{p-1}\right)$. In the $(p+q)$-dimensional theory, there are solutions with both magnetic and electric charge under the field strength $F_{q}$, so there is flux quantization, and we can ask how $c_{\text {thermo }}$ scales with $N$, the number of flux quanta through the compact space. For $\operatorname{AdS}_{p} \times \mathrm{S}^{q}$, we can reason out this scaling by recalling that in an asymptotically flat solution, the number of branes enters the harmonic function in the metric as $H=1$ $+c_{1} N\left(l_{\mathrm{Pl}} / r\right)^{q-1}$, where $c_{1}$ is some dimensionless constant. Thus $L$ and $R$ scale as $N^{1 /(q-1)} l_{\mathrm{Pl}}$. In a near-extremal solution, the Bekenstein-Hawking entropy scales as $\left(L / l_{\mathrm{Pl}}\right)^{p+q-2}$, whereas the Hawking temperature does not scale with $l_{\mathrm{Pl}}$ at all. Putting everything together, one finds

$$
c_{\text {thermo }} \sim N^{(p+q-2) /(q-1)} .
$$

This specializes to the odd results $c_{\text {thermo }} \sim N^{25 / 22}$ for $\mathrm{AdS}_{4} \times \mathrm{S}^{23}$ and $c_{\text {thermo }} \sim N^{25 / 3}$ for $\mathrm{AdS}_{23} \times \mathrm{S}^{4}$. These peculiar fractions do not bring any known CFT's to mind, but at least they represent something to shoot for in constructing putative duals of $\operatorname{AdS}_{p} \times \mathrm{M}_{q}$.

\footnotetext{
${ }^{6}$ We thank O. Bergman and A. Brandhuber for discussions on these and related points.
} 


\section{IMPLICATIONS FOR EXTREMAL BLACK BRANES AND NEGATIVE ENERGY}

As mentioned above, the (nondilatonic) theories of gravity (2.1) all contain charged black brane solutions, where the charge is obtained by integrating $\mathrm{F}_{q}$ over an $\mathrm{Sj}^{q}$ surrounding the brane. (For the general solution, see [12].) In particular, there are extremal black branes, with metric

$$
d s^{2}=H^{-2 /(p+1)}\left(-d t^{2}+d \mathbf{y} \cdot d \mathbf{y}\right)+H^{2 /(q-1)}\left(d r^{2}+r^{2} d \Omega_{q}\right)
$$

where $H$ is the harmonic function $H(r)=1+c_{1} N\left(l_{\mathrm{Pl}} / r\right)^{q-1}$. The near horizon limit is just $\operatorname{AdS}_{p} \times \mathrm{S}^{q}$. So the stability we have found for $\operatorname{AdS}_{p} \times \mathrm{S}^{q}$ for all $p$ and $q$ is consistent with the expected stability of extremal solutions. However, we have also seen that $\mathrm{AdS}_{p} \times \mathrm{M}_{n} \times \mathrm{M}_{q-n}$ is unstable, when $q$ $<9$ and $\mathrm{M}_{n}, \quad \mathrm{M}_{q-n}$ are Einstein spaces. These can also arise as the near horizon limit of a type of extremal black brane as follows. Consider the cone over $\mathrm{M}_{n} \times \mathrm{M}_{q-n}$

$$
d s^{2}=d r^{2}+r^{2}\left(d \sigma_{M_{n}}^{2}+d \sigma_{M_{q-n}}^{2}\right) .
$$

This space is Ricci flat, and has a curvature singularity at the apex $r=0$. (Even though the curvature goes to zero for large $r$, this space is not asymptotically flat in the usual sense since the curvature only falls off like $r^{-2}$.) Suppose one places a stack of branes at the apex of the cone, extended in the orthogonal directions. The resulting exact solution is obtained by simply replacing the flat transverse metric in Eq. (11.1) with the cone metric (11.2).

One might have expected this new solution to be stable, since it is the extremal limit of a family of black brane solutions. However it is easy to see that it is not (at least for $q<9)$. The near horizon limit is now $\operatorname{AdS}_{p} \times \mathrm{M}_{n} \times \mathrm{M}_{q-n}$ which is unstable to a perturbation [Eq. (8.5)] that goes to zero asymptotically in $\mathrm{AdS}_{p}$. So a similar perturbation with support very close to the horizon of the extremal black brane will also grow exponentially. This is independent of the change in boundary conditions at infinity since, in the Poincaré coordinates appropriate to the near horizon geometry of $\mathrm{AdS}_{p}$, a scalar field near the horizon has a unique evolution inside a spacetime region that includes infinite Poincaré time. One might object that extremal black branes are always unstable in the sense that adding a small amount of energy causes them to become nonextremal, ${ }^{7}$ and the horizon moves from an infinite distance to a finite distance (in spacelike directions). However, as we will see, our perturbation is very different in that it can actually decrease the mass.

A natural question to ask is what does this instability lead to? As we have seen, the unstable mode causes one factor, say $\mathrm{M}_{n}$, to shrink in size and the other to grow. So one might expect that in the full nonlinear evolution, $\mathbf{M}_{n}$ simply shrinks to zero size. However this cannot happen. It has recently been shown [22] that if the weak energy condition is satisfied, event horizons cannot have collapsing cycles. In fact,

\footnotetext{
${ }^{7}$ This is true for branes of finite extent. For infinite branes, one needs nonzero energy density to become nonextremal.
}

given any spacelike curve on the event horizon, if one evolves the curve along the null geodesic generators, its length cannot go to zero in finite affine parameter. The basic idea is to use the fact that the divergence $\theta$ of the null geodesic generators $l$ of the event horizon cannot become negative. This means that if part of the horizon is contracting, the orthogonal directions must be expanding. But this introduces a lot of shear $\sigma_{M N}$ in the null geodesic congruence. One now uses the Raychaudhuri equation

$$
\frac{d \theta}{d \lambda}=-\frac{\theta^{2}}{D-2}-\sigma_{M N} \sigma^{M N}-R_{M N} l^{M} l^{N}
$$

where $\lambda$ is an affine parameter along the null geodesics and $D$ is the total spacetime dimension. If the weak energy condition is satisfied, the right hand side is negative definite, so when the shear becomes large, $\theta$ decreases rapidly. One can show that if part of the horizon shrinks to zero size in finite affine parameter, $\theta$ must become negative which is a contradiction. So the solution must settle down to a new static configuration. In [22], this result was discussed in the context of the Gregory-Laflamme instability of nonextremal black branes. In that case, the horizon starts to shrink in some places and expand in others, and it was widely believed that the horizon would eventually pinch off and form separate black holes. However this cannot happen. Instead, the solution must settle down to a new static black brane solution without translational symmetry along the brane.

The instability we are discussing can be viewed as an extremal analog of the Gregory-Laflamme instability. Since our theory satisfies the weak energy condition, and the result in [22] does not require that the horizon is nonextremal, it can also be applied to our case. Thus, $\mathbf{M}_{n}$ cannot shrink to zero size, and there must be another static solution whose near horizon geometry is not $\mathrm{AdS}_{p} \times \mathrm{M}_{n} \times \mathrm{M}_{q-n}{ }^{8}$

Strictly speaking, the near horizon limit of the black brane solution includes only part of $\mathrm{AdS}_{p}$ (the region covered by the Poincaré coordinates). Suppose we now consider the global solution $\operatorname{AdS}_{p} \times \mathrm{M}_{n} \times \mathrm{M}_{q-n}$ and ask what happens if we perturb it in the unstable direction. As a first step toward answering this question, we show that there are solutions in the full nonlinear theory which are asymptotically $\mathrm{AdS}_{p} \times \mathrm{M}_{n} \times \mathrm{M}_{q-n}$ and have arbitrarily negative energy (where, as usual, we measure energy relative to $\mathrm{AdS}_{p}$ ). Since the perturbation violates the $\mathrm{BF}$ bound, it is clear we can lower the energy slightly by turning on this mode. To show the energy can be arbitrarily negative, it suffices to construct suitable initial data. Consider the spatial metric

\footnotetext{
${ }^{8}$ One might worry that there will be a problem applying the result in [22] since the unstable extremal black brane is not asymptotically flat in the usual sense. However, even though null infinity is not well defined, one can still define the event horizon as the boundary of the past of a surface at large $r$, and the result will still apply.
} 


$$
\begin{aligned}
d s^{2}= & {\left[\frac{r^{2}}{L^{2}}+1-\frac{m(r)}{r^{p-3}}\right]^{-1} d r^{2}+r^{2} d \Omega_{p-2}+e^{(q-n) \phi(r)} d \sigma_{M_{n}} } \\
& +e^{-n \phi(r)} d \sigma_{M_{q-n}}
\end{aligned}
$$

so $m=0, \phi=0$ corresponds to the metric on a static surface (in global coordinates) for $\operatorname{AdS}_{p} \times \mathrm{M}_{n} \times \mathrm{M}_{q-n}$. The total mass is proportional to $m(\infty)$. Notice that the volume of the $q$-dimensional internal space is independent of $\phi$. This is a nonlinear generalization of the perturbation we considered in Sec. VIII. We again set $\mathrm{F}_{q}=c \operatorname{vol}_{M_{q}}$. If we set all time derivatives to zero, the only constraint on this initial data is the Hamiltonian constraint of general relativity which implies that the scalar curvature of Eq. (11.4) must be $c^{2} / 2$ where $c^{2}$ is given by Eq. (2.8). This yields a first order differential equation which can be used to solve for $m(r)$ in terms of $\phi(r)$. If we assume $\phi$ is everywhere small, this equation becomes

$$
\frac{m^{\prime}}{r^{p-2}} \propto\left[\frac{r^{2}}{L^{2}}+1-\frac{m(r)}{r^{p-3}}\right]\left(\phi^{\prime}\right)^{2}-\frac{2(p-1)^{2}}{(q-1) L^{2}} \phi^{2} .
$$

The right-hand side resembles the energy density of the linearized unstable mode (8.5) except that the $\phi^{\prime}$ term involves the corrected spatial metric. Since the term involving $m(r)$ on the right hand side only decreases the energy density we can get an upper limit on the mass by dropping it. One can now explicitly find $\phi(r)$ so that $m(\infty)$ is arbitrarily negative. For example, if $q<9-(8 / p)$, one can take $\phi=\phi_{0} e^{-r / a}$. The total mass is negative for large $a$, and goes to minus infinity as $a \rightarrow \infty$.

If we start with $\operatorname{AdS}_{p} \times \mathrm{M}_{n} \times \mathrm{M}_{q-n}$ and perturb it slightly, the energy will be only slightly negative. As we have just seen, this is very far from the minimum energy solution. A priori, one might expect $\mathrm{M}_{n}$ to collapse down to zero size in finite time. This will produce a curvature singularity. It is unlikely that this singularity is naked, since we don't expect cosmic censorship to be violated so easily in the higher dimensional theory of gravity we are considering. It may form a black hole, or in light of the horizon results, $\mathrm{M}_{n}$ may not collapse down at all. In the latter case, since we are using reflecting boundary conditions at infinity (appropriate for the AdS-CFT correspondence), the solution may not settle down to any static configuration. It would be interesting to investigate this further.

We have not considered the massive type IIA theory in this section. It would also be interesting to investigate the implications of the instability of $\mathrm{AdS}_{4} \times \mathrm{S}^{6}$ for negative energies and extremal black branes in this theory.

\section{ACKNOWLEDGMENTS}

We would like to thank O. Bergman, I. Klebanov, and E. Witten for useful discussions. We are particularly grateful to A. Brandhuber for help checking some of the equations in the massive type IIA compactifications. The work of S.S.G. and I.M. was supported in part by the DOE under grant DEFG03-92ER40701 and through the Outstanding Junior Inves- tigator program. I.M. was also supported under grant DEFG02-91ER40671. The work of O.D. was supported by the NSF under grant PHY-99-07949. The work of G.H. was supported by the NSF under grant PHY-00-70895. The work of D.Z.F. was supported by the NSF under grant PHY-9722072. I.M. thanks the Institute for Theoretical Physics, Santa Barbara and the Particle Theory Group at Caltech for their hospitality during most of the phases of this project.

\section{APPENDIX}

Here we collect conventions and a few properties of the differential operators we employ. We work in a metric of signature $(-++\cdots+)$ and define the Ricci tensor in terms of the Riemann tensor by $R_{M N} \equiv R_{M P N}^{P}$.

The Hodge-de Rham Laplacian $\Delta_{y}=-\left(d^{\dagger} d+d d^{\dagger}\right)$ is negative-definite, but in the case of a compact Riemannian Einstein space of positive curvature a more stringent bound can be derived for the case of one-forms. We use $-R^{2} \Delta_{y} Y^{I} \equiv \kappa^{I} Y^{I}$, and for the ordinary Laplacian $\square_{y}$ $\equiv g^{\alpha \beta} \nabla_{\alpha} \nabla_{\beta},-R^{2} \square_{y} Y^{I} \equiv \lambda^{I} Y^{I}$. For scalar spherical harmonics $Y^{I}, \square_{y}=\Delta_{y}$, and a vanishing eigenvalue always exists corresponding to $Y^{I}=$ const. $^{9}$ For one-forms, we may consider

$$
\begin{aligned}
0 & \leqslant \int\left(\nabla^{\alpha} Y^{I \beta}+\nabla^{\beta} Y^{I \alpha}\right)\left(\nabla_{\alpha} Y_{\beta}^{I}+\nabla_{\beta} Y_{\alpha}^{I}\right) \\
& =2 \int \nabla^{\alpha} Y^{I \beta}\left(\nabla_{\alpha} Y_{\beta}^{I}+\nabla_{\beta} Y_{\alpha}^{I}\right) \\
& =-2 \int Y^{I \beta}\left(\square_{y}+\frac{q-1}{R^{2}}\right) Y_{\beta}^{I} \\
& =-2 \int Y^{I \beta}\left(\Delta_{y}+\frac{2(q-1)}{R^{2}}\right) Y_{\beta}^{I},
\end{aligned}
$$

proving $\kappa^{I} \geqslant 2(q-1)$; furthermore, equality occurs for $\left(\nabla_{\alpha} Y_{\beta}^{I}+\nabla_{\beta} Y_{\alpha}^{I}\right)=0$, which is the condition for $Y_{\beta}^{I}$ to be a Killing vector. Additionally, the absence of harmonic oneforms $Y_{\alpha}^{h}$ on a compact Einstein space of positive curvature may be proved as follows. Any harmonic one-form must satisfy $\nabla^{\alpha} Y_{\alpha}^{h}=0=\nabla_{\alpha} Y_{\beta}^{h}-\nabla_{\beta} Y_{\alpha}^{h}$, so

$$
\begin{aligned}
0 & =\int \nabla^{\alpha} Y^{h \beta}\left(\nabla_{\alpha} Y_{\beta}^{h}-\nabla_{\beta} Y_{\alpha}^{h}\right) \\
& =\int\left(\nabla^{\alpha} Y^{h \beta} \nabla_{\alpha} Y_{\beta}^{h}+\frac{q-1}{R^{2}} Y^{h \beta} Y_{\beta}^{h}\right),
\end{aligned}
$$

which is impossible as the right-hand side is a sum of a nonnegative and a positive quantity.

For the case of $S^{q}$, the eigenvalues $\lambda^{I}$ of the ordinary Laplacian $\square_{y}$ for the various tensor harmonics are

\footnotetext{
${ }^{9}$ One can derive the bound $\lambda^{I} \geqslant q$ for nonconstant $Y^{I}[14]$.
} 


\begin{tabular}{llc} 
Tensor harmonic & \multicolumn{1}{c}{$\lambda^{I}$} & Range of $k$ \\
\hline$Y^{I}$ & $k(k+q-1)$ & $k \geqslant 0$ \\
$Y_{\alpha}^{I}$ & $k(k+q-1)-1$ & $k \geqslant 1$ \\
$Y_{\left[\alpha_{1} \cdots \alpha_{n}\right]}^{I}$ & $k(k+q-1)-n$ & $k \geqslant 1$ \\
$Y_{(\alpha \beta)}^{I}$ & $k(k+q-1)-2$ & $k \geqslant 2$
\end{tabular}

while for the Hodge-de Rham Laplacian acting on vectors, we obtain

$$
\kappa^{I}=(k+1)(k+q-2), \quad k \geqslant 1 .
$$

[1] J. Maldacena, Adv. Theor. Math. Phys. 2, 231 (1998); Int. J. Theor. Phys. 38, 231 (1998).

[2] S. S. Gubser, I. R. Klebanov, and A. M. Polyakov, Phys. Lett. B 428, 105 (1998).

[3] E. Witten, Adv. Theor. Math. Phys. 2, 253 (1998).

[4] O. Aharony, S. S. Gubser, J. Maldacena, H. Ooguri, and Y. Oz, Phys. Rep. 323, 183 (2000).

[5] P. G. Freund and M. A. Rubin, Phys. Lett. 97B, 233 (1980).

[6] P. Breitenlohner and D. Z. Freedman, Phys. Lett. 115B, 197 (1982).

[7] A. Adams and E. Silverstein, Phys. Rev. D 64, 086001 (2001).

[8] M. J. Duff, B. E. Nilsson, and C. N. Pope, Phys. Lett. 139B, 154 (1984).

[9] M. Berkooz and S. Rey, J. High Energy Phys. 01, 014 (1999); Phys. Lett. B 449, 014 (1999).

[10] S. Kachru and E. Silverstein, Phys. Rev. Lett. 80, 4855 (1998).

[11] G. T. Horowitz and L. Susskind, "Bosonic M theory," hep-th/0012037.

[12] G. W. Gibbons, G. T. Horowitz, and P. K. Townsend, Class. Quantum Grav. 12, 297 (1995).
[13] L. J. Romans, Phys. Lett. 169B, 374 (1986).

[14] M. J. Duff, B. E. Nilsson, and C. N. Pope, Phys. Rep. 130, 1 (1986).

[15] L. Castellani, R. D’Auria, P. Fre, K. Pilch, and P. van Nieuwenhuizen, Class. Quantum Grav. 1, 339 (1984).

[16] H. J. Kim, L. J. Romans, and P. van Nieuwenhuizen, Phys. Rev. D 32, 389 (1985).

[17] P. van Nieuwenhuizen, Class. Quantum Grav. 2, 1 (1985).

[18] B. Biran, A. Casher, F. Englert, M. Rooman, and P. Spindel, Phys. Lett. 134B, 179 (1984).

[19] L. Mezincescu and P. K. Townsend, Ann. Phys. (N.Y.) 160, 406 (1985).

[20] P. Breitenlohner and D. Z. Freedman, Ann. Phys. (N.Y.) 144, 249 (1982).

[21] P. K. Townsend, Phys. Lett. 148B, 55 (1984).

[22] G. Horowitz and K. Maeda (unpublished).

[23] A. Brandhuber and Y. Oz, Phys. Lett. B 460, 307 (1999).

[24] J. Polchinski, String Theory (Cambridge University Press, Cambridge, England, 1988), Vol. 2.

[25] I. R. Klebanov and E. Witten, Nucl. Phys. B556, 89 (1999). 